\title{
Influence of structural modifications of automotive brake systems for squeal events with kriging meta-modelling method
}

\author{
Enora Denimal ${ }^{1,2, *}$, Jean-Jacques Sinou $^{2,3}$, and Samuel Nacivet ${ }^{2}$ \\ ${ }^{1}$ Laboratoire de Tribologie et Dynamique des Systèmes, UMR CNRS 5513, École Centrale de Lyon, \\ 36 avenue Guy de Collongue 69134 Écully Cedex, France \\ ${ }^{2}$ PSA Peugeot Citroën, Centre technique de la Garenne Colombes, 18 rue des Fauvelles, \\ 92250 La Garenne Colombes, France \\ ${ }^{3}$ Institut Universitaire de France, 75005 Paris, France \\ ${ }^{*}$ Corresponding authors: enora.denimal@inria.fr ; jean-jacques.sinou@ec-lyon.fr
}

\begin{abstract}
Squeal noise is an important issue in the automotive industry since it is one of the main reasons for the return of vehicles to the customer service. Hence, it is essential to predict it in the design stage of a brake system. The Complex Eigenvalue Analysis (CEA) remains the most widely used method to predict squeal noise. The numerical cost associated to this method is important enough to make impossible the use of parametric studies. The present study proposes the use of the kriging method to surrogate the eigenvalues computed by the CEA by taking into consideration different uncertain parameters, namely the friction coefficient and two small masses added to the caliper that correspond to a classical choice of structural modifications used in the final phase of a brake design to avoid squeal noise. Thus, it is possible to assess the influence of the structural modifications on the stability of the brake system and to get information to choose the best brake design. Finally, uncertainty propagation is performed to get a robust design of the brake.
\end{abstract}

Keywords Squeal noise, Complex Eigenvalue Analysis, Finite Element Model, Kriging, Meta-modelling, Uncertainty propagation

\section{Introduction}

In the automotive industry, squeal noise is one of the main reasons for the return of vehicles to the customer services, representing high costs. Indeed, in the collective imagination this noise is associated to a technical failure of the brake system. For this reason, squeal noise is predicted early in the design stage to avoid it.

Hence, squeal has been a subject of research for a few decades. The first studies were dedicated to the mechanisms that originate squeal. In a first time, tribological origins were considered with the stick-slip phenomenon [1]. Then Spurr [2] proposed a model where the vibrations come from a geometrical modification of the system and finally the concept of mode coupling appears which explains the squeal instability by the coupling between two modes of the structure because of friction. By means of phenomenological models [3, 4], one can demonstrate that the friction brings non-symmetric terms in the stiffness matrix bringing complex eigenvalues and so instabilities may appear for some values of the friction coefficient. Hence, an increase of the friction coefficient may lead to a mode coupling phenomenon. In other words, two frequencies merge while the two associated real parts of the eigenvalues bifurcate. When one of those becomes positive, the system becomes unstable. This analysis, called CEA for Complex Eigenvalue Analysis, is based on the determination of the eigenvalues of the system around its non-linear static sliding equilibrium. However, this analysis may lead to an under- or over-estimation of the number modes involved in the non-linear dynamic response of the brake system and cannot predict all the complexity of the non-linear signature of transient and stationary squeal events [5]. Indeed, a dynamic transient analysis has to be perform in addition to the stability analysis to predict the non-linear behaviour of the squeal events [6-9]. However, the time integration methods or the alternative non-linear methods may have a high computational cost and need complex implementation which make them 
unaffordable or difficult to use in an industrial context. For these reasons, the CEA remains the privileged method in an industrial context, and more particularly in the automotive industry.

Deterministic codes incite a one input - one output vision where the parameters are often summarized to their mean values. For very influential parameters, some parametric studies might be considered. However, the consideration of uncertainty associated to some parameters may improve significantly the prediction of squeal [10]. Before considering the propagation of the uncertainty, it is necessary to identify it and to quantify it. Different kinds of uncertainty exist such as the uncertain parameters, for which the exact value is unknown; the parametric variables, which can be design parameters; the model bias, since the equations are an approximation; etc. More generally, uncertainties are classified into two classes: aleatory and epistemic. An aleatory uncertainty corresponds to an uncertainty related to the inherent character of the variable considered. This kind of uncertainty is often described by a Probability Density Function (PDF). The epistemic uncertainty is due to a lack of knowledge.

Hence, statistical approaches have been considered to study brake squeal. Numerically, probabilistic or nonprobabilistic methods can be used to consider uncertainty. Monte Carlo Simulations (MCS) have been conducted in a first time [11] to propagate the uncertainty of the contact instability. Because the MCS strategy is quite inefficient, other strategies have been considered such as first-order perturbation method [12] or Polynomial Chaos Expansion (PCE) [13]. Whilst these methods provide interesting results, they require a high number of computation and are not always adapted to a large number of parameters. Thus, developments on the use of meta-modelling methods have been found since recently with the use of the linear regression [14-16], neural networks or the kriging method [17, 18]. Nobari et al. [17], used kriging to predict the evolution of the eigenvalue of one unstable mode of an automotive brake system by considering the uncertainty associated to the Young modulus of different components and the friction coefficient. Nechak et al. [18] proposed to used kriging to study the impact of four uncertain parameters (namely the Young modulus of both the pad and disc, the friction coefficient and the contact stiffness at the friction interface between pad and disc) on the squeal propensity for a simplified brake system. The authors are then interested only in the study of instability due to friction from a global point of view. However, none deep study is performed to discuss the drawbacks and the difficulties of using meta-models for prediction of the occurrences of brake systems subjected to multiinstabilities. The problem of meta-modelling with multi-instabilities (with a high number of unstable modes and not only one or two instabilities) can be a difficult subject because of the presence of many unstable modes in a restricted frequency range and of the possibility of crossing phenomenon between the stable and unstable modes. This makes it difficult to track the evolution of each mode versus the evolution of physical parameters. Indeed, the construction of relevant and representative meta-models and tracking the evolution of each unstable mode versus the evolution of physical parameters can be very difficult from a numerical point of view. So the originality of this paper is not only to propose a deep discussion and a complete study on this subject for an automotive brake system with multi-contact interfaces, but also to propose a new specific methodology to correctly use meta-modelling for an industrial brake system for which many unstable modes may appear in the frequency range of interest. The ultimate goal is to be able to use meta-models to bring a deep understanding and a whole description of the global stability behaviour of the brake system as well as the evolution of each unstable mode in the design interval of interest. To demonstrate the feasibility of the proposed strategy different types of uncertain parameters will be considered in the present study. They correspond to structural modifications conventionally used in the automotive industry to reduce brake squeal. Thus, the objective of the meta-modelling will be to assess the impact of each structural modification on each unstable mode and more generally on the propensity of brake squeal. More concretely, the methodology presented here will be tested to discuss the design of an automotive brake system regarding squeal.

The paper is organized as follows. First, the finite element model of the automotive brake system under study is presented as well as the numerical strategy used for the squeal prediction and the different uncertain parameters. Then, the theoretical aspects of kriging will be given, and the global strategy applied. In the third part of the study, some difficulties related to the creation of the experimental design are highlighted and the new adopted strategy presented. Finally, the kriging methodology is used to create the meta-models. They are first validated and then used to study the impact of the different parameters on the stability of the system. Uncertainty propagation is then performed to assess a robust description of the stability behaviour of the system.

\section{Presentation of the mechanical system under study}

This section is devoted to the presentation of the Finite Element Model (FEM) of the automotive brake system under study as well as the numerical strategy adopted for the prediction of squeal occurrences. Finally, the different uncertain parameters used for validating the efficiency of the kriging methodology are presented. 


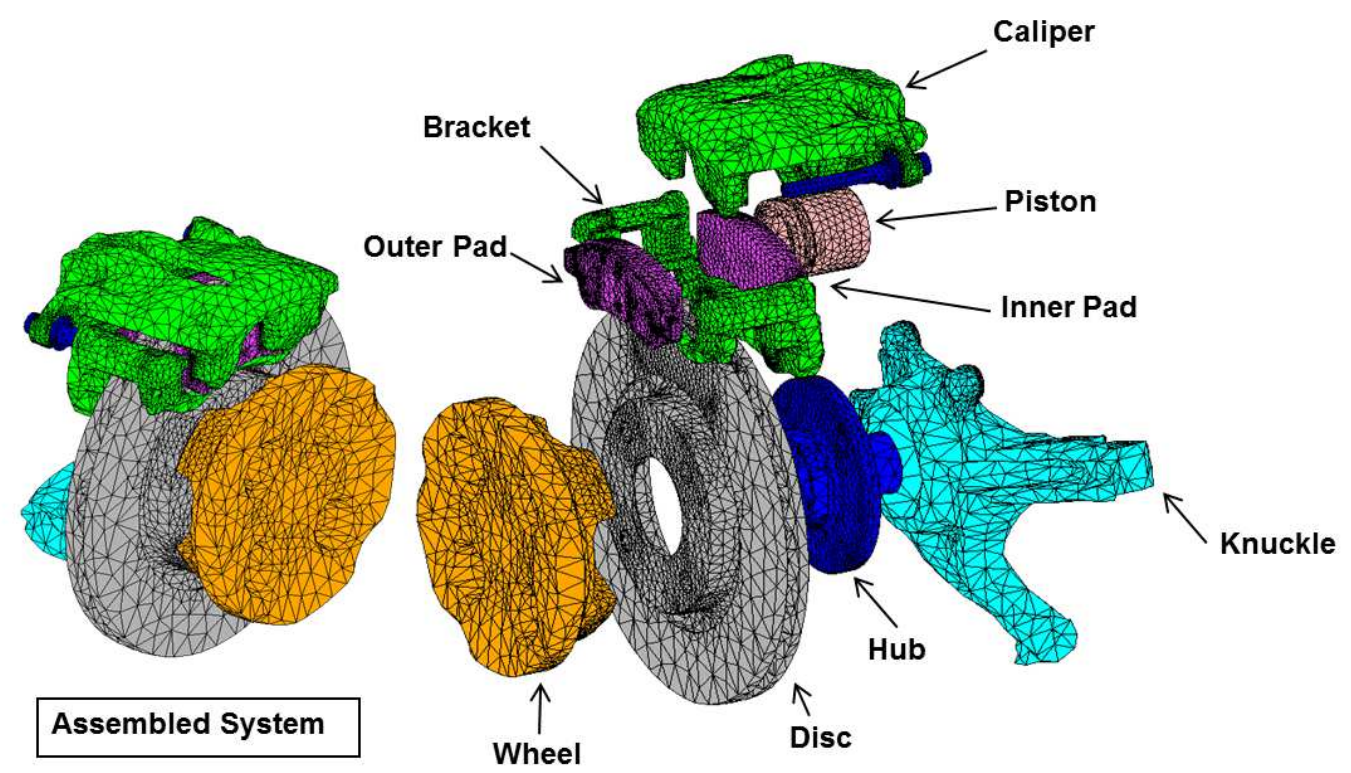

Figure 1: View of the Finite Element Model of the brake developed with Abaqus - Assembled view (left) and exploded view (right)

\begin{tabular}{llccc}
\hline Element & Material & Young Modulus $(\mathbf{G P a})$ & Poisson's coefficient & Density $\left(\mathrm{kg} / \mathbf{m}^{3}\right)$ \\
\hline Disc & Grey cast iron & 113 & 0.275 & 7075 \\
Caliper & Ductile cast iron & 175 & 0.275 & 7200 \\
Bracket & Ductile cast iron & 175 & 0.275 & 7200 \\
Pad (support) & Steel & 210 & 0.29 & 7800 \\
Brake lining & GA7504 & 10.66 & 0.09 & 4870 \\
& & 10.66 & 0.14 & \\
\hline
\end{tabular}

Table 1: Material properties of the elements of the brake system

\subsection{The finite element model of the brake system}

The system under study is displayed Figure 1. It corresponds to an automotive brake system with a floating caliper technology. The main components are the disc, the floating caliper, the outer and inner pads, the hub knuckle, the piston and the wheel. A braking action consists in the following steps: a hydraulic pressure is applied on the piston during the breaking action and the inner pad is pushed until it touches the disc. Then, the reaction forces pull back the caliper and so the outer pad against the opposite side of the disc. This action creates friction between the pads and the disc. During this event, brake squeal may appear and even if it does not affect the braking efficiency, it is highly unpleasant for the user.

The implementation of the FEM is performed on Abaqus. A volume mesh is realized with 10-node quadratic tetrahedron, it represents 103,444 elements and 182,337 nodes. The FEM is validated with a convergence study with regards to the eigenvalues and mode shapes. The latter is not presented here for the sake of concision. The material properties of the different components of the brake system are given in Table 1, the brake lining is an orthotropic material so each line corresponds to the characteristics of the material in one direction. A classical Coulomb's law is used for the frictional interfaces between the pads and the disc and a linear penalty method is used for the contact constraint enforcement. Braking pressure and disc rotation velocity are constant, and the hydraulic pressure is modelled with an effort applied on the back of the piston and on the caliper surface. The dynamic equation of the brake system can be written as :

$$
\mathbf{M} \ddot{\mathbf{X}}+\mathbf{C} \dot{\mathbf{X}}+\mathbf{K X}+\mathbf{F}_{\mathbf{n l}}(\mathbf{X})=\mathbf{F}_{\text {ext }}
$$

where $\mathbf{M}, \mathbf{K}$ and $\mathbf{C}$ are the mass, stiffness and damping matrices without contact and friction at the pads/disc interfaces, respectively. $\mathbf{X}$ defines the vector of the generalized displacements and the dot denotes the derivative with respect to the time. The vector $\mathbf{F}_{\text {ext }}$ corresponds to the external efforts applied on the brake 
system, namely the pressure applied on the piston and the caliper. The vector $\mathbf{F}_{\mathbf{n l}}$ represents the contribution of both the non-linear contact forces and frictional forces at the pad/disc interfaces. At this stage of the paper it is important to underline the fact that nine different contact interfaces of internal and external pads are also considered for the automotive brake system under study (in addition to the pad/disc interfaces). The complete description of the FEM brake system as well as the influence of various contact interfaces between pads, piston, bracket and caliper are not the objective of the present study. The interested reader can refer for more details in [19].

\subsection{Numerical prediction of the squeal propensity: Complex Eigenvalue Analysis}

As previously explained, the CEA is the method mainly used to predict squeal in the automotive industry because of its low numerical cost compare to the other methods despite its over- or under-predictive nature of the unstable modes. It consists in the determination of the eigenvalues of the brake system around its non-linear sliding equilibrium since they characterize the possible appearance of an initial growing oscillatory behaviour.

The full CEA procedure is briefly detailed below. The first step of the CEA consists in the determination of the non-linear static sliding equilibrium $\mathbf{U}_{\mathbf{S}}$ of the system (i.e. $\mathbf{K U}_{\mathbf{S}}+\mathbf{F}_{\mathbf{n l}} \mathbf{U}_{\mathbf{S}}=\mathbf{F}_{\mathbf{e x t}}$ ). Then, the local stability is studied by considering a small perturbation around this equilibrium position. The associated eigenvalues problem is defined by:

$$
\left(\lambda^{2} \mathbf{M}+\lambda \mathbf{C}+\left(\mathbf{K}+\mathbf{J}_{\mathbf{n l}}\right)\right) \mathbf{\Psi}=\mathbf{0}
$$

where $\mathbf{J}_{\mathbf{n}}$ denotes the Jacobian matrix of the non-linear forces $\mathbf{F}_{\mathbf{n l}}$ at the point $\mathbf{U}_{\mathbf{S}}$. It is worth to note that the non-linear contribution of the two pad/disc interfaces as well as the nine different contact interfaces of internal and external pads are considered (see [19] for more details). $\lambda$ is an eigenvalue and $\boldsymbol{\Psi}$ the associated eigenvector. The global stiffness matrix (i.e. $\mathbf{K}+\mathbf{J}_{\mathbf{n l}}$ ) becomes non-symmetric due to the friction terms in $\mathbf{J}_{\mathbf{n l}}$, so the eigenvalues are complex as well as the eigenvectors. Considering the $j^{t h}$ eigenvalue $\lambda_{j}=a_{j}+i \omega_{j}$ and the associated eigenvector $\boldsymbol{\Psi}_{j}, \omega_{j}$ defines the pulsation of the $\mathrm{j}^{\text {th }}$ mode and $a_{j}$ the associated real part. If all the real parts of eigenvalues are negative, then the local equilibrium point is considered as stable but if at least one of them is positive, then the local equilibrium point is considered as unstable. It is worth to notice that the real part $a_{j}$ corresponds to the initial growth rate of the dynamic response due to the appearance of one instability. So the higher is the real part, the higher is the growth rate of the dynamic response of the perturbed system while the system remains in the neighbourhood of the equilibrium position.

\subsection{Uncertain parameters of the brake system}

In the design of an automotive brake system, the robustness against brake squeal must be validated. It is admitted that the friction coefficient at the pad/disc interface $\mu$ has a strong influence on the stability of the system. For this reason, in the design stage, the stability of the brake system is investigated for different values of this friction coefficient. When a brake system appears to have a too high squeal propensity, structural modifications are investigated to cope with this issue. In the automotive industry, the classical first way to reduce brake squeal is to investigate the effect of different designs of pads, calipers or discs on the squeal propensity. Sometimes, the idea is to make very small modifications on an existing brake system, without design modification of the different components of the brake system. This second choice generally resides in a concern to find a solution to reduce brake squeal for a brake system that is already on the market or in the final phase of conception, so that it is impossible to do a new complete design of all the brake components. In this second case, the structural modifications can be the addition of small masses on different components of the system for example. This choice will be proposed to illustrate the present study and the efficiency of the kriging methodology in this context.

Thereby, three uncertain parameters are considered in the present study. The first one corresponds to the friction coefficient $\mu$ at the pads/disc interfaces. More precisely two modelling choices will be made on this parameter. Initially, studies will be conducted for a fixed value of the friction coefficient ranging from 0 to 1 in order to get information on the whole stability behaviour of the brake system (Sections 4 and 5.3). In a second time, different probability laws for the friction coefficient will be considered. This second choice of modelling is based on the fact that the friction can vary between two braking actions because of the environment and so it is unknown and can be described with an experimental distribution law [20]. This will be described in more detail in the Section 5.4.

The two other parameters correspond to design parameters and more precisely to the addition of two small masses on two opposite sides of the brake caliper. Here they are modelled with a modification of the material density of two volumes displayed in red and blue in Figure 2. It corresponds to an addition of mass ranging between $0 \mathrm{~g}$ and $250 \mathrm{~g}$ on each side and they are denoted $M_{1}$ (red one) and $M_{2}$ (blue one). The consideration 


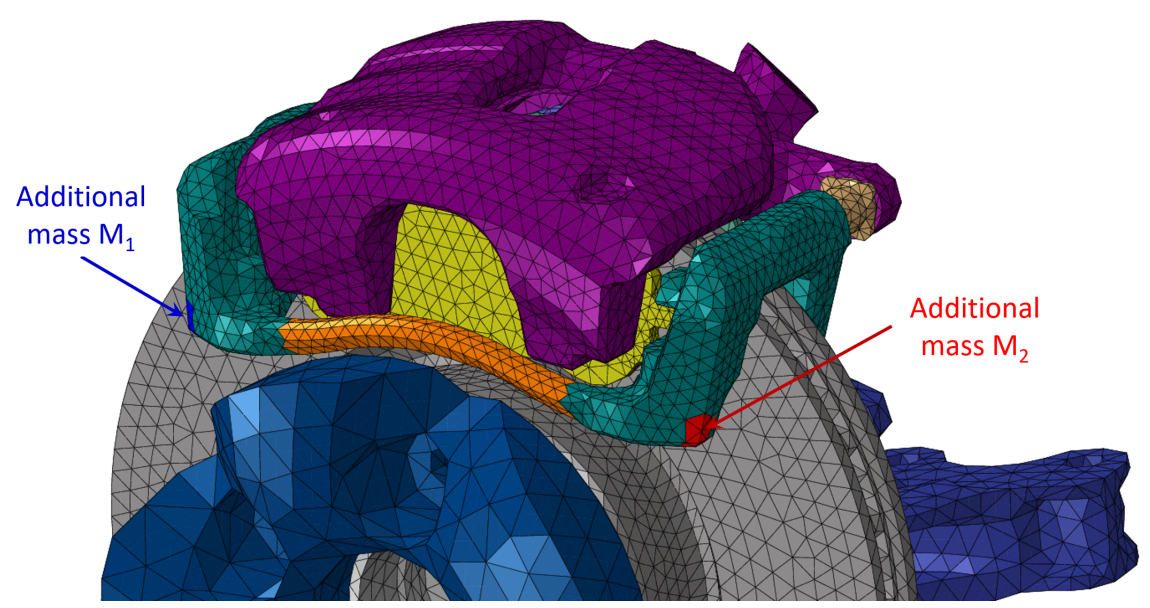

Figure 2: Structural modification of the brake system

\begin{tabular}{lclcc}
\hline Parameter & Notation & Nature & Min. value & Max value \\
\hline Friction coefficient & $\mu$ & random & 0 & 1 \\
Mass 1 & $M_{1}$ & parametric & $0 \mathrm{~g}$ & $250 \mathrm{~g}$ \\
Mass 2 & $M_{2}$ & parametric & $0 \mathrm{~g}$ & $250 \mathrm{~g}$ \\
\hline
\end{tabular}

Table 2: Uncertain parameters

of these two parameters comes from different reasons. First, from an industrial point of view, they correspond to two design parameters used to improve the efficiency of a brake with respect to squeal. Usually, a maximum of $250 \mathrm{~g}$ in total is added but in this study, it is possible to consider that all the mass is concentrated on one side. Then, from a scientific point of view, these two masses have a strong influence on the mode shapes of the caliper which is often involved in the mode coupling phenomena and so on the stability behaviour of the brake system, as it will be demonstrated in Section 4. Thus, this set of parameters will make it possible to verify the effectiveness of the kriging method on a difficult and complex example in relation to the strong variability of squeal frequencies (i.e. unstable modes) in relation to the parameters considered. The different uncertain parameters and their characteristics are summarized Table 2.

\section{The kriging methodology}

A strategy based on meta-modelling method consists in the approximation of a function with a mathematical model that is constructed with a set of input/output determined with the complete model. Once the mathematical model is constructed, it simulates the behaviour of the complete model and for a same input, it gives the same output.

In this section, the mathematical aspects of kriging are quickly presented. For more information, the reader can refer to [21-24].

\subsection{Mathematical formulation}

Kriging is an interpolation method where interpolated values are modelled by a Gaussian process. In other words, the objective is to create a function $\mathcal{M}$ between an input vector $\mathbf{x}$ and an output scalar $y$. If the dimension of the output is superior to 1 , then a kriging model is build for each dimension. To create a surrogate model, a set of inputs/outputs $\left\{\left(\mathbf{x}^{(i)}, y_{i}\right)_{i=1, \ldots, N}\right\}$, called Experimental Design (ED), is generated by the user. The kriging approach consists in approximating the real process by a surrogate process $\mathcal{M}$ written as:

$$
\mathcal{M}(\mathbf{x})=\sum_{j=1}^{q} g_{j}(\mathbf{x}) \beta_{j}+\mathcal{Z}(\mathbf{x})
$$

where the $\left(g_{j}\right)_{j=1, \ldots, q}$ are $q$ known real-valued functions, $\boldsymbol{\beta}$ is a vector of coefficients to be determined and $\mathcal{Z}$ is a stationary Gaussian process of zero mean. Its covariance function is given by $C\left(\mathbf{x}, \mathbf{x}^{\prime}\right)=\sigma^{2} \mathcal{R}\left(\mathbf{x}, \mathbf{x}^{\prime}\right)$ where $\sigma^{2}$ 


\begin{tabular}{|c|c|}
\hline Kernel & $\mathrm{k}(\mathrm{d}, \boldsymbol{\theta})$ \\
\hline Gaussian & $k(d, \theta)=\exp \left(-\frac{d^{2}}{2 \theta^{2}}\right)$ \\
\hline Exponential & $k(d, \theta)=\exp \left(-\frac{|d|}{\theta}\right)$ \\
\hline General exponential & $k(d, \theta)=\exp \left(-\left(\frac{|d|}{-\theta}\right)^{p}\right)$ \\
\hline Matérn $\nu=5 / 2$ & $k(d, \theta)=\left(1+\frac{\sqrt{5}|d|}{\theta}+\frac{5 d^{2}}{3 \theta^{2}}\right) \exp \left(-\frac{\sqrt{5}|d|}{\theta}\right)$ \\
\hline Matérn $\nu=3 / 2$ & $k(d, \theta)=\left(1+\frac{\sqrt{3}|d|}{\theta}\right) \exp \left(-\frac{\sqrt{3}|d|}{\theta}\right)$ \\
\hline Linear & $k(d, \theta)=\max \left(0,1-\frac{|d|}{\theta}\right)$ \\
\hline Spherical & $k(d, \theta)=\left\{\begin{array}{l}1-\frac{3}{2} \frac{|d|}{\theta}+\frac{1}{2}\left(\frac{|d|}{\theta}\right)^{3}, \text { if }|d|<\theta \\
0, \text { otherwise }\end{array}\right.$ \\
\hline Cubic & $k(d, \theta)=\left\{\begin{array}{l}1-3\left(\frac{|d|}{\theta}\right)^{2}+2\left(\frac{|d|}{\theta}\right)^{3}, \text { if }|d|<\theta \\
0, \text { otherwise }\end{array}\right.$ \\
\hline Spline & $k(d, \theta)=\left\{\begin{array}{l}1-15\left(\frac{|d|}{\theta}\right)^{2}+30\left(\frac{|d|}{\theta}\right)^{3}, \text { if } 0 \leq \frac{|d|}{\theta}<0.2 \\
1.25\left(1-\frac{|d|}{\theta}\right)^{3}, \text { if } 0.2<\frac{|d|}{\theta}<1 \\
0, \text { otherwise }\end{array}\right.$ \\
\hline
\end{tabular}

Table 3: Example of covariance functions in dimension 1

is the unknown variance of the process and $\mathcal{R}\left(\mathbf{x}, \mathbf{x}^{\prime}\right)$ is the correlation function which is known. The functions $\left(g_{j}\right)_{j=1, \ldots, q}$ are chosen according to the a priori knowledge of the phenomenon to model, they are often taken as polynomial functions of order 0,1 or 2 . Usually, the function $\mathcal{R}$ is unknown but is often constructed with a family of kernel functions parametrized by a parameter $\boldsymbol{\theta}$.

By assuming $\mathcal{Z}$ as a Gaussian process, then the best unbiased predictor is linear. The regression matrix $\mathbf{G}$ is defined as the matrix $G_{i j}=g_{j}\left(\mathbf{x}^{(i)}\right)$ and the correlation matrix $\mathbf{R}$ is given by $\left.R_{i j}=C\left(\mathbf{x}^{(i)}, \mathbf{x}^{(j)}\right)\right)$. If one wants to predict the response $y_{0}$ at the point $\mathbf{x}_{0}$, then $\mathbf{g}_{0}$ is defined as the vector of $g_{j}\left(\mathbf{x}_{0}\right)$ and $\mathbf{r}_{0}$ as the vector of $C\left(\mathbf{x}_{0}, \mathbf{x}^{(i)}\right)$. The predictor of $\mathbf{x}_{0}$ is given by:

$$
\mathcal{M}\left(\mathbf{x}_{0}\right)=y_{0}=\mathbf{g}_{0}^{\mathrm{T}} \tilde{\boldsymbol{\beta}}+\mathbf{r}_{0}^{\mathrm{T}} \mathbf{R}^{-1}(\mathbf{y}-\mathbf{G} \tilde{\boldsymbol{\beta}})
$$

where $\tilde{\boldsymbol{\beta}}=\left(\mathbf{G}^{\mathrm{T}} \mathbf{R}^{-1} \mathbf{G}\right)^{-1} \mathbf{G}^{\mathrm{T}} \mathbf{R}^{-1} \mathbf{y}$ and the mean square error is:

$$
\operatorname{MSE}\left(\mathbf{x}_{0}\right)=\sigma^{2}\left(1-\mathbf{g}_{0}^{\mathrm{T}} \mathbf{R}^{-1} \mathbf{g}_{0}+\left(\mathbf{g}_{0}-\mathbf{G}^{\mathrm{T}} \mathbf{R}^{-1} \mathbf{r}_{0}\right)^{\mathrm{T}}\left(\mathbf{G}^{\mathrm{T}} \mathbf{R}^{-1} \mathbf{G}\right)^{-1}\left(\mathbf{g}_{0}-\mathbf{G}^{\mathrm{T}} \mathbf{R}^{-1} \mathbf{r}_{0}\right)\right)
$$

The reader can notice that the predictions are exact at the design points and that the variance is zero at these points. It is also interesting to notice that the variance depends only of the inputs and not of the outputs.

The choice of the correlation function is crucial in the creation of the kriging meta-model and previous developments are only valid if the matrix $\mathbf{R}$ is positive-definite. A classical way to construct it, is to choose a kernel function family parametrized by $\boldsymbol{\theta}$ to be determined from the available data by maximizing the likelihood function or by minimizing the cross-validation error. Stationary kernel are often considered, i.e. they depends from $\left(\mathbf{x}-\mathbf{x}^{\prime}\right)$. In dimension 1 , several families are available, some examples are given Table 3 . If the dimension is superior to 1 , then the tensorial product of these functions is considered, so:

$$
\mathcal{R}\left(\mathbf{x}, \mathbf{x}^{\prime}\right)=\prod_{j=1}^{d} k\left(d_{j}, \theta_{j}\right)
$$

where $d_{j}=\mathbf{x}_{j}-\mathbf{x}_{j}^{\prime}$ and $d$ the dimension of the input space. The $\theta_{j}$ must be determined. The estimation of $\boldsymbol{\theta}$ is performed by solving a complex optimization problem and different algorithm can be used [21, 22, 24].

\subsection{Global strategy}

The global strategy is the following: 
1. the input set of the experimental design is generated with an optimized Latin Hypercube Sampling (LHS) in order to have a homogeneous repartition of the points in the space,

2. the corresponding CEA are performed,

3. each eigenvalue is tracked with respect to the evolution of the different input parameters to generate the different output sets of the experimental design,

4. for each eigenvalue, two meta-models are created: one on the real part and one on the frequency,

5. the different meta-models are validated,

6. the meta-models are used to analyse the stability of the brake system assuming a variation of the parameters and uncertainty propagation is performed for random parameters.

To performed these different steps, two packages of $\mathrm{R}$ are used: DiceDesign, for the creation of the experimental design and DiceKriging for the creation and the validation of the different meta-models [21]. The different steps of kriging as well as the main drawbacks and difficulties of use in the context of an industrial brake system subjected to multi-instabilities are developed in the following.

\section{Creation of the Experimental Design (ED) and discussion on the difficulty of the mode tracking}

Some developments about the creation of the experimental design are given here. Indeed, once the input set is generated and the associated CEA performed, it is then necessary to track each eigenvalue in order to create their own experimental design. According to previous works made on a phenomenological model in [25], a MAC criterion can be used to track the evolution of the different mode shapes with respect to the evolution of the design parameters, this step is crucial to create the different experimental design for each eigenvalue. However, the industrial system presented here involves a large number of modes compared to the phenomenological model, making the system more complex. The aim of this part is first to discuss on the difficulty of the mode tracking based on the MAC criterion and then to extend the strategy proposed in [25] to the industrial brake system.

\subsection{Preamble - illustration on the difficulty of the use of mode tracking}

For this study, only the friction coefficient $\mu$ at the pad/disc interfaces is retained as variable. The two additional masses $M_{1}$ and $M_{2}$ are zero. This choice is based on the fact that the influence of the friction coefficient on the stability behaviour of the brake system is of first order. Therefore, this preliminary study will help answer the question of whether it is possible to track the variation of each mode of the system when one parameter varies. In the following, a CEA is performed for a range of friction coefficient values from 0.05 to 0.9 with a step of 0.05. Nine mode couplings are observed, as illustrated in Figure 4. In the following the focus is put on the mode coupling that appears at $\mu=0.55$ for a frequency equals to $1118 \mathrm{~Hz}$. Thanks to the small scanning step, it is possible to track "by hand" the evolution of the two modes before the coalescence and the appearance of the instability. In the following, the mode 1 will denote the stable mode for which the real part becomes strictly negative and whose frequency varies between $1080 \mathrm{~Hz}$ and $1120 \mathrm{~Hz}$. The mode 2 will denote the unstable mode for which the real part becomes strictly positive and whose frequency varies between $1170 \mathrm{~Hz}$ and $1100 \mathrm{~Hz}$.

The strategy presented in [25] consists in tracking the eigenvectors associated to the eigenvalues by comparing two sets of mode shapes computed in $\mathbf{p}$ and in $\mathbf{p}+\boldsymbol{\Delta} \mathbf{p}$ where $\mathbf{p}$ is the vector of the input parameters and $\boldsymbol{\Delta} \mathbf{p}$ a small variation of it. The comparison between the mode shapes is realized with a MAC criterion and paired are made from the largest values. In order to test this strategy on the FEM, the different MAC matrices obtained by comparing the mode shapes of the modes 1 and 2 between $\mu$ and $\mu+\Delta \mu$ are determined. At this level, all the points of the model are used to compute the MAC. The different MAC matrices are given Figure 4. It is possible to notice that before the coalescence (i.e. before $\mu=0.5$ ), the extra-diagonal terms of the different MAC matrices are low. It means the mode shapes of the two modes are very different. However, the closer the coalescence is, the higher the extra-diagonal terms are, and they become equal to 1 at the bifurcation point. Indeed, the MAC matrix between the mode shapes at $\mu=0.5$ and $\mu=0.55$ is only composed of 1 . After the coalescence, the extra-diagonal values decrease a little until 0.75 which means the mode shapes of the two coupling modes are similar. In other words, it is impossible to distinguish the two modes not only after the coalescence pattern but also at the bifurcation point. For example, in the case considered here, when $\mu=0.1$, the mode shape of the mode 2 is a mode that involves the caliper and the outer pad whereas the mode shape 1 involves only the disc. After the bifurcation, the two modes are visually identical and involves only the caliper and the outer pad. 


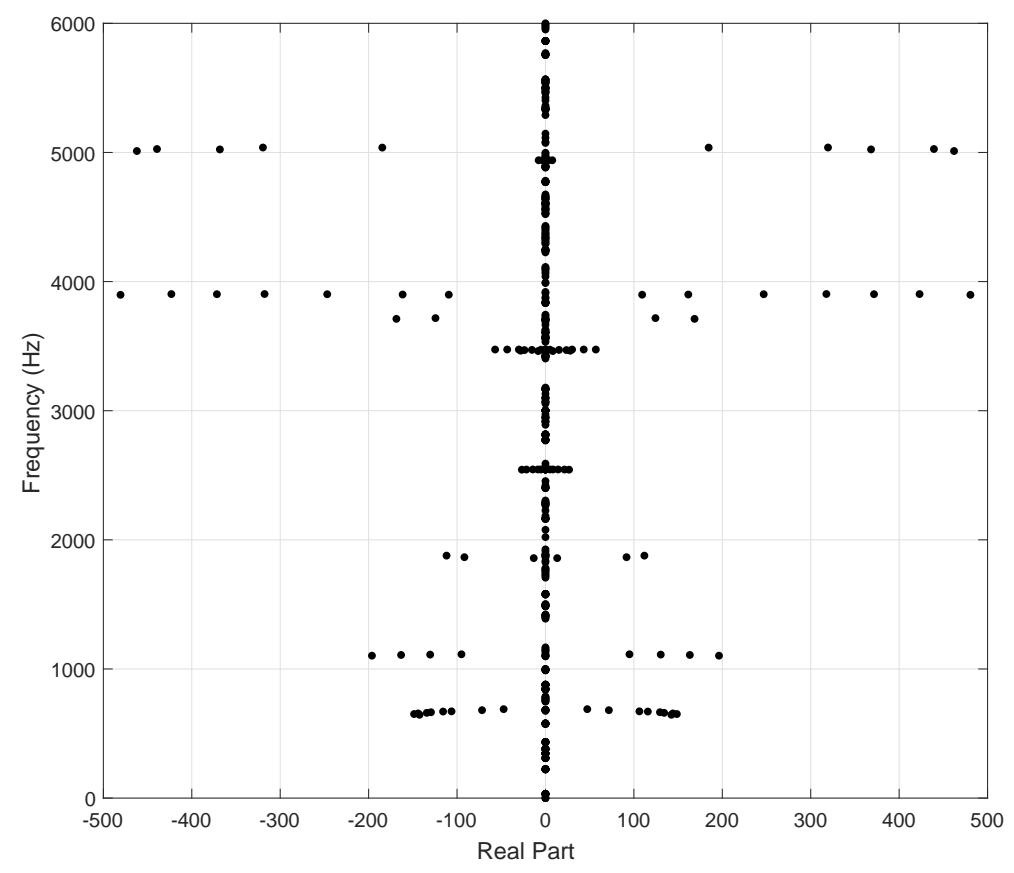

Figure 3: Eigenvalues for different values of the friction coefficient $(\mu \in[0.05,1])$ in the complex plan
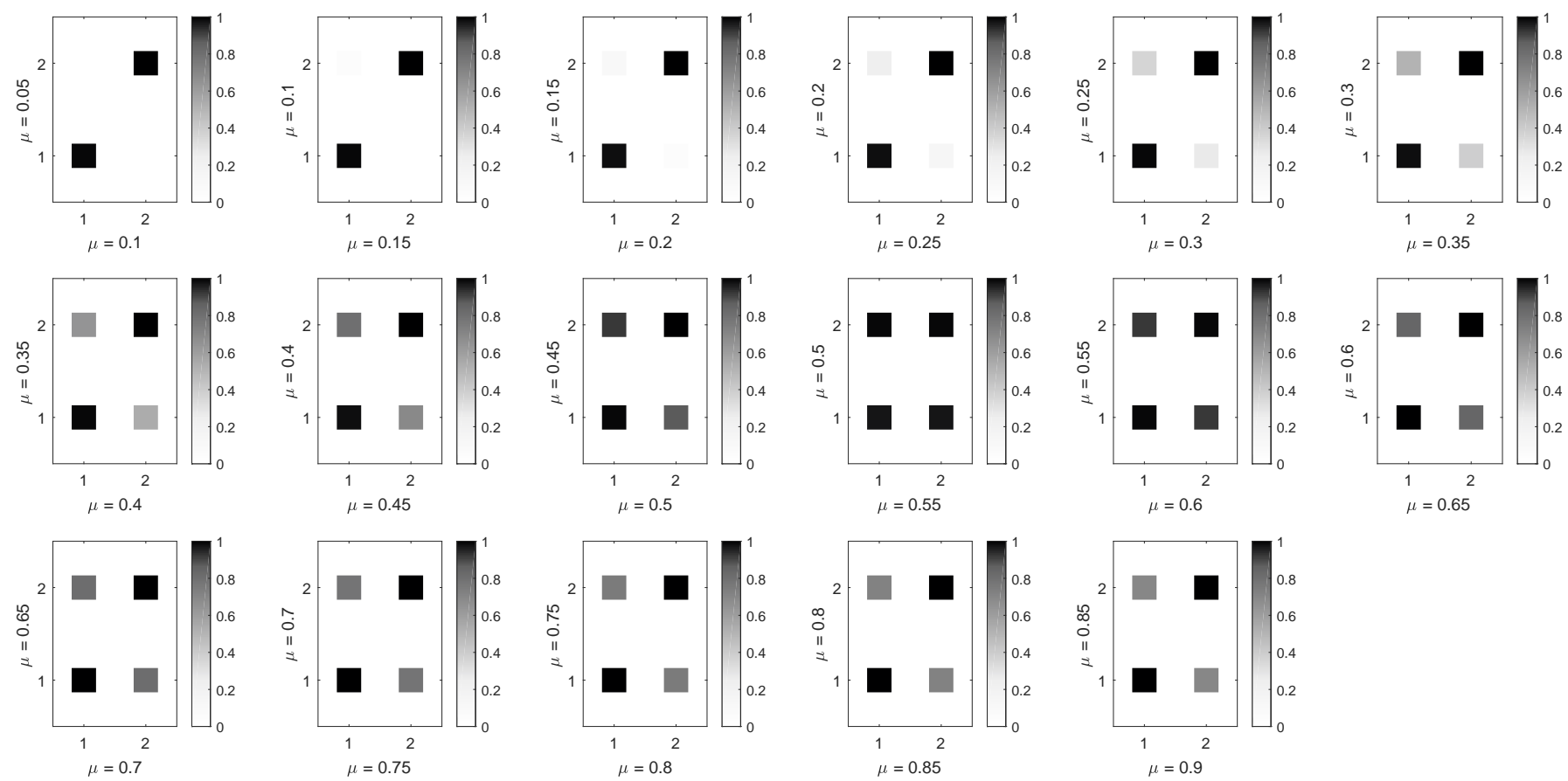

Figure 4: Evolution of the different MAC matrices between the mode shapes of 1 and 2 between the successive values of $\mu$ 


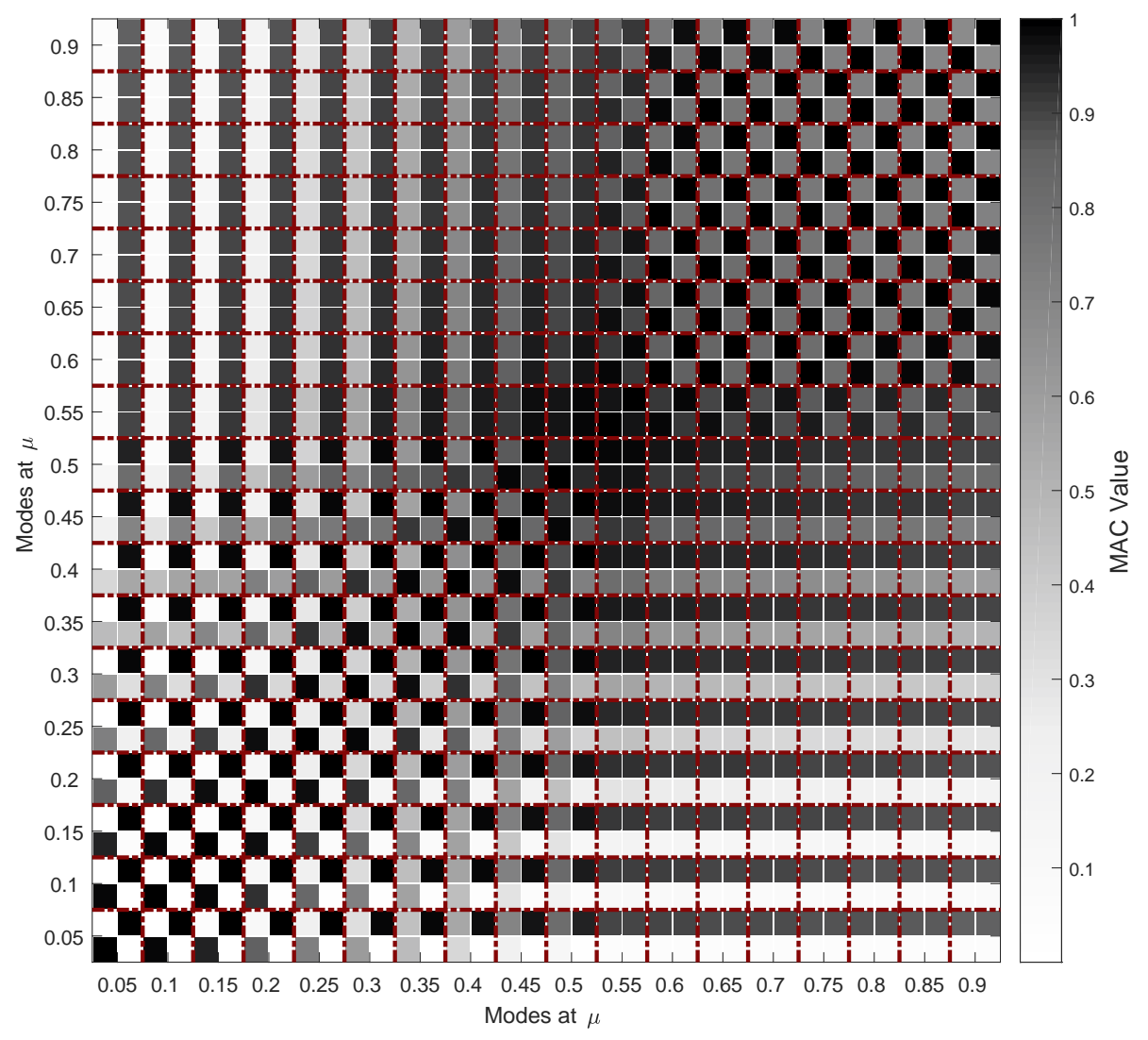

Figure 5: AutoMAC matrices of the different modes shapes of mode 1 and 2 for the different values of the friction coefficient $\mu$

The last important point is the choice of the step for the friction coefficient $\mu$. Indeed, a a small scan step was chosen to get minor variations of the mode shapes between two points to track them. However, the objective is to have the ED with as few points as possible to have the lowest possible cost. It is then necessary to find the maximal step on $\mu$ that allows to track the eigenvectors. In order to evaluate the influence of the step of discretization on $\mu$, the different mode shapes of the modes 1 and 2 are concatenated and the AutoMAC matrix is determined. It is displayed Figure 5. Red lines correspond to the separation between the different MAC matrices for the different values of $\mu$. Hence, the block on line $i$ and column $j$ corresponds to the MAC matrix of the mode shapes obtained for the $i^{\text {th }}$ value of $\mu$ and the modes obtained for the $j^{\text {th }}$ value of $\mu$. The blocks on the diagonal are the different AutoMAC matrices and the blocks just under or over the diagonal are the different MAC matrices determined for two successive values of $\mu$, in other words the matrices presented previously Figure 4. The first observation that can be made is that the MAC matrix of the bloc $(\mu=0.55$, $\mu=0.55)$ is only composed of MAC values equal to the unity, so the mode shapes are identical. Moreover, according to the evolution of the blocks located on the first row, only the mode 2 has its mode shape at $\mu=0.05$ that correlates with mode shapes observed after the coalescence. Whereas, the mode 1 has a mode shape at $\mu=0.05$ that does not correlate with mode shapes observed after the coalescence. Hence, the two coupled modes have, after the coalescence, a mode shape that is similar to only one of the two mode shapes observed before the coalescence. Moreover, it appears that after the coalescence, by comparing the MAC matrices for $\mu>0.55$, the diagonal terms are always really closed to 1 whatever the variation on $\mu$. The extra-diagonals terms are high but inferior to 1 which makes possible to differentiate the two modes. However, by comparing the two first blocks of the bottom line for $\mu<0.55$, it can be noticed that a too high variation of $\mu$ implies a decrease in the extra-diagonal terms up to 0.3 or even 0.1 for one of the two modes (see the block $(\mu=0.05$, $\mu=0.5)$ ). The choice of the step on the friction coefficient must be done in order to ensure the tracking of the mode shape, and so can not be too large. This conclusion is partly incompatible with considerations of pre-design study via kriging methods for which the discretization of each design variables cannot be chosen as refined.

These different remarks and observations prove that the mode tracking with a MAC criterion seems to be a complicated task in an industrial context for a system with a large number of potential unstable modes. Indeed, it would have been preferable to be able to build an ED of a small size to have a low global numerical cost for 
the construction of the meta-models. However, a too small number of points makes it more difficult to track the modes, particularly in the coalescence zone. In fact, a high discretization in this area should be necessary, more particularly on $\mu$ because of the high sensitivity to this parameter. This strategy is a dead-end since it implies adding too many points in each coalescence zones, which can be numerous and distinct.

Other strategies have been investigated based on the study of the sub-vector space generated by the different unstable mode shapes as proposed in [26, 27] but no better conclusion appears and the two coupled complex modes seem to tend toward the same real mode. Because the mode shapes are highly sensitive with regards to a controlled parameter (i.e. the friction coefficient $\mu$ in the present case), the mode tracking step will be a tedious task since the MAC criterion will be quickly deteriorated. The choice of the ED and its size is of major importance to ensure a correct mode tracking. Moreover, because it is not possible to track the two real modes behind the mode coupling, the final strategy will be a restriction of the strategy proposed in [25] and the mode shapes will be tracked only when the real parts of the eigenvalues are non-zero. The initial objective was to characterize the system when it is unstable, so this restriction is not in contradiction with this objective. Consequently, the meta-models will be constructed only on the unstable configurations of the brake system. From an industrial point of view, this amounts to focusing on meta-modelling and predicting only squeal events (i.e. the propensity of brake squeal), without seeking to predict the behaviour of the brake system when it is stable. This restrictive objective is in perfect agreement with the expectations of engineers of the automotive industry on the subject of brake squeal.

\subsection{New strategy}

Finally, the results presented here are obtained with all the points of the finite element model for the mode shapes. But for storage reasons, this strategy is not relevant for the ED creation. Hence, a set of 500 points homogeneously distributed on the system is retained. They are chosen to minimize the extra-diagonal terms of the AutoMAC matrix of the unstable modes on a configuration of the system.

The new strategy consists in the unstable mode tracking when one instability appears for a specific chosen frequency range. In the following, the part of the design space where at least one unstable mode appears will be refereed by the term unstable zone. It is worth noting that damping was neglected in this study for a better understanding and investigating of the proposed methodology. However, all the proposed analysis and the kriging methodology based on the restriction of meta-modelling of unstable modes (i.e. modes with a positive real part) is also applicable without any additional difficulty if damping is added.

For each eigenvalue (so for each mode), two meta-models will be created from this unstable zone. One for the real part, denoted $\mathcal{M}_{a}$, and one for the frequency, denoted $\mathcal{M}_{\omega}$. However, this unstable zone does not correspond to a regular Cartesian product of intervals of the design space, so it is necessary to get a test function that tells if the two meta-models $\mathcal{M}_{a}$ and $\mathcal{M}_{\omega}$ are in their validity zone or not. To perform this, a new ED is considered for the real part. On the points of the total ED where no pairing was performed, the value 0 is assigned for the real part. This can be justified by the fact that the mode was tracked when it is unstable, and so, on the other points of the total ED, the mode is stable (i.e. the real part of the eigenvalue is zero). From this new ED, a third meta-model is created, denoted $\mathcal{M}_{a 0}$. If for an input, the absolute value of the output $\mathcal{M}_{a 0}$ is superior to a threshold, then the meta-models $\mathcal{M}_{a}$ and $\mathcal{M}_{\omega}$ are valid and can be used. It is important to notice that the attribution of a zero value where no pairing was performed can be dangerous. Indeed, the mode tracking strategy can "miss" a few points and these points does not have to be assigned to a zero value. To avoid this problem, the different ED are always checked and aberrant points are removed.

\section{Application for the squeal prediction}

In this section the relevance and effectiveness of the proposed methodology is investigated for the prediction of squeal propensity on an industrial brake system. As previously explained explained in Section 2.3 three uncertain parameters are considered in the present study: the first one corresponds to the friction coefficient $\mu$ at the pads/disc interfaces and the two other parameters correspond to the addition of two small masses $M_{1}$ and $M_{2}$ on two opposite sides of the brake caliper as illustrated in Figure 2. The associated characteristics for each uncertain parameter are given in Table 2 can vary.

This section is organized as follows: first, the different experimental designs for the unstable modes are created. Then, the different meta-models are created and validated. Finally, the meta-models are used to analyse the influence of the different parameters on the stability of the system and to perform uncertainty propagation. 


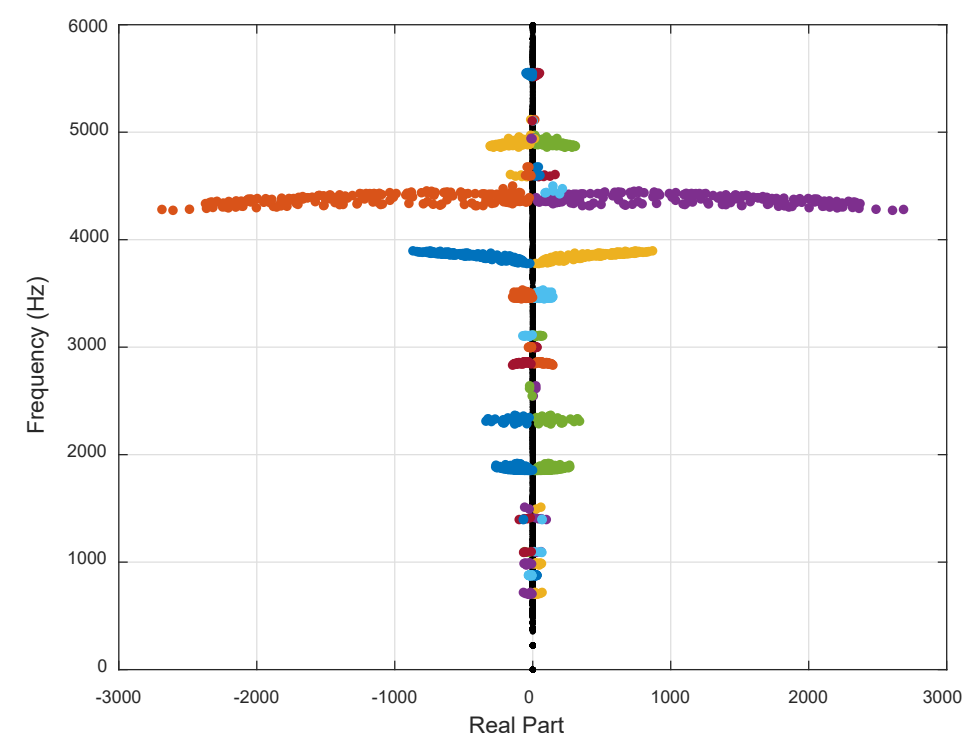

Figure 6: Experimental design after mode tracking

\subsection{Creation of the experimental design}

To generate the input points of the ED, an optimized Latin Hypercube Sampling (LHS) is created [21]. The points are regularly distributed in the design space which increase the precision of the meta-model [28]. 250 points are so generated, and the interval boundaries are also added (i.e. 8 additional points). This leads to a final input set of the ED with 258 points. The corresponding CEA are performed, and a MAC criterion is used to distinguished the different mode shapes in the unstable zones as explained previously. The results are displayed Figure 6 where the different eigenvalues are represented in the complex plan. Each color corresponds to the evolution of an unstable or a stable mode for a specific mode coupling. It can be noted that the use of a MAC criterion makes it possible to differentiate between the different modes of the brake system as illustrated around $4500 \mathrm{~Hz}$ where several mode coupling are present.

Moreover, a set of 50 points, denoted validation set, is generated as reference results. This validation set will be used to perform comparisons between the reference results and the kriging predictions in order to discuss and demonstrate the relevance of the proposed strategy. It is worth to notice that each mode has its own experimental design according to the part of the space where the real part of its eigenvalue is non-zero. For each mode, the different ED are observed and aberrant points removed as previously explained in Section 4.

Finally, the kriging strategy will be used for nine coupling modes. The retained stable and unstable modes are summarized Table 4 where the maximum of the real part, the associated frequency, the number of points available for each ED and the number of validation points are given. It is important to note that only modes with a sufficiently large number of points are retained (i.e. with more than 25 points in the ED) to build metamodels with enough learning points. This fact corresponds to one of the limitations of using a meta-modelling strategy. In other words, if one instability is not sufficiently well described in the design space via the input points of the ED (i.e. the number of input points of the ED for which the instability appears is small enough) then the latter will not be considered for the study. From an industrial point of view, this implies that the propensity of brake squeal to such instability in the design space is low enough to be neglected.

\subsection{Creation of the kriging meta-models and validation}

For each mode, the three meta-models $\mathcal{M}_{a}, \mathcal{M}_{\omega}$ and $\mathcal{M}_{a 0}$, previously defined in Section 4.2, are created. The package DiceKriging available on the software $\mathrm{R}$ is used to create the different meta-models [21]. Two important choices must be made by the user to build efficient meta-models: the type of regression function and the family of kernel functions for the covariance determination. Three types of polynomial regression are considered here, namely the 0 order, 1 st order and 2 nd order with the first interaction term. Three types of kernel are considered here, namely Matérn 5/2, Matérn 3/2 and exponential kernel. As previously seen in [25], the best combination differs according to the eigenvalue so for each meta-model, the combination of regression function and kernel function that minimizes the Leave-One-Out (LOO) error is chosen. The latter is explained in the following with the validation indicators. 


\begin{tabular}{cccccc}
\hline Mode $\mathbf{n}^{o}$ & Nature & Real part & Frequency $\mathbf{( H z )}$ & Number of ED points & Number of validation points \\
\hline 1 & Unstable & 30.4 & 877.5 & 27 & 7 \\
2 & Unstable & 268.5 & 1885.5 & 129 & 24 \\
3 & Unstable & 339.1 & 2311.7 & 29 & 4 \\
4 & Unstable & 146.0 & 2832.8 & 47 & 9 \\
5 & Unstable & 145.8 & 3468.9 & 203 & 36 \\
6 & Unstable & 868.2 & 3896.3 & 186 & 34 \\
7 & Unstable & 2687.4 & 4281.3 & 215 & 42 \\
8 & Unstable & 309.0 & 4871.7 & 128 & 19 \\
9 & Unstable & 48.9 & 5550.4 & 26 & 5 \\
10 & Stable & -30.4 & 877.5 & 27 & 7 \\
11 & Stable & -268.5 & 1885.5 & 129 & 24 \\
12 & Stable & -339.1 & 2311.7 & 30 & 4 \\
13 & Stable & -146.0 & 2832.8 & 47 & 9 \\
14 & Stable & -145.8 & 3468.9 & 203 & 38 \\
15 & Stable & -868.2 & 3896.3 & 186 & 34 \\
16 & Stable & -2687.4 & 4281.3 & 215 & 42 \\
17 & Stable & -309.0 & 4871.7 & 128 & 19 \\
18 & Stable & -48.9 & 5550.4 & 26 & 5 \\
\hline
\end{tabular}

Table 4: Characteristics of the main modes

Once the different meta-models are constructed, it is important to validate them. Two strategies are adopted here. The first one is based on the comparison of the kriging prediction to the reference values from the validation set. This approach has the advantage of being simple to understand and intuitive. However, it has the disadvantage of requiring additional sample points that are not used for the construction of the meta-models. This leads to the need for additional calculations, which is not in line with a strategy to reduce calculation times. In order to avoid this, a second strategy will be used to validate the different meta-models without additional points. This strategy is based on three classical indicators [21] to validate meta-models only from the data used (without the need of additional validation points) will be used and explained in a second time.

As a first strategy of validation, the prediction of the kriging meta-models $\mathcal{M}_{a}$ and $\mathcal{M}_{\omega}$ are compared to reference values of the validation set. The strategy is the following, a set of 50 points is generated on the whole input space and the corresponding CEA are performed. Thanks to a MAC criterion, the different modes are tracked. But as previously explained, this tracking process is only possible when the real parts of the eigenvalues are different from zero. Thus, each mode has its own validation set with a different number of validation points. Then, for each mode, the prediction of the kriging meta-models $\mathcal{M}_{a}$ and $\mathcal{M}_{\omega}$ are compared to the prediction of the reference values of its validation set. Results are displayed Figure 7 for the real parts and for the frequencies. Kriging predictions are in orange and reference CEA results in blue. For each mode, the predictions are really close to the reference results even when strong variation are observed as for the frequency of mode 7 or for the real part of modes 5 and 2 for example. If this way of validating the relevance of the meta-models has the advantage to give visual results that are easy to understand and help to have a confidence in the meta-models, it requires the evaluation of additional points that might be computationally expensive. Indeed, a CEA calculation takes in average $45 \mathrm{~min}$ on the whole model. If a 50-points validation set is considered, then about 37 hours are required for the generation of the validation set.

Thereby, three classical indicators [21] based on the calculated statistics will be now briefly described and used to validate the relevance of the kriging meta-models. These indicators serve as diagnostics to indicate whether the meta-modelling and its associated parameter values are relevant and reasonable. In other words, they give some idea of how well the meta-model predicts the values at unknown locations before producing the final surface by using only the points that have already been computed for the creation of the meta-models. They do not require the use of additional computations, as is the case for the first strategy of validation. More specifically, these indicators will be illustrated on the meta-models of the real part of modes 4 and 6 , the results being similar for the other meta-models. The first indicator is based on the Leave-One-Out (LOO) strategy. The LOO method is a classical method used to validate a meta-model without additional point. This validation removes part of the data, classically call the test dataset, and uses the rest of the data, classically called the training dataset, to validate the relevance of the kriging meta-models by using the trend and autocorrelation models to be used for prediction. Thereby, as a validation procedure, the LOO might be used as follow for each point $i$ of the experimental design. A new meta-model $\mathcal{M}_{\sim i}$ is built from the ED without the point $i$. Then, 

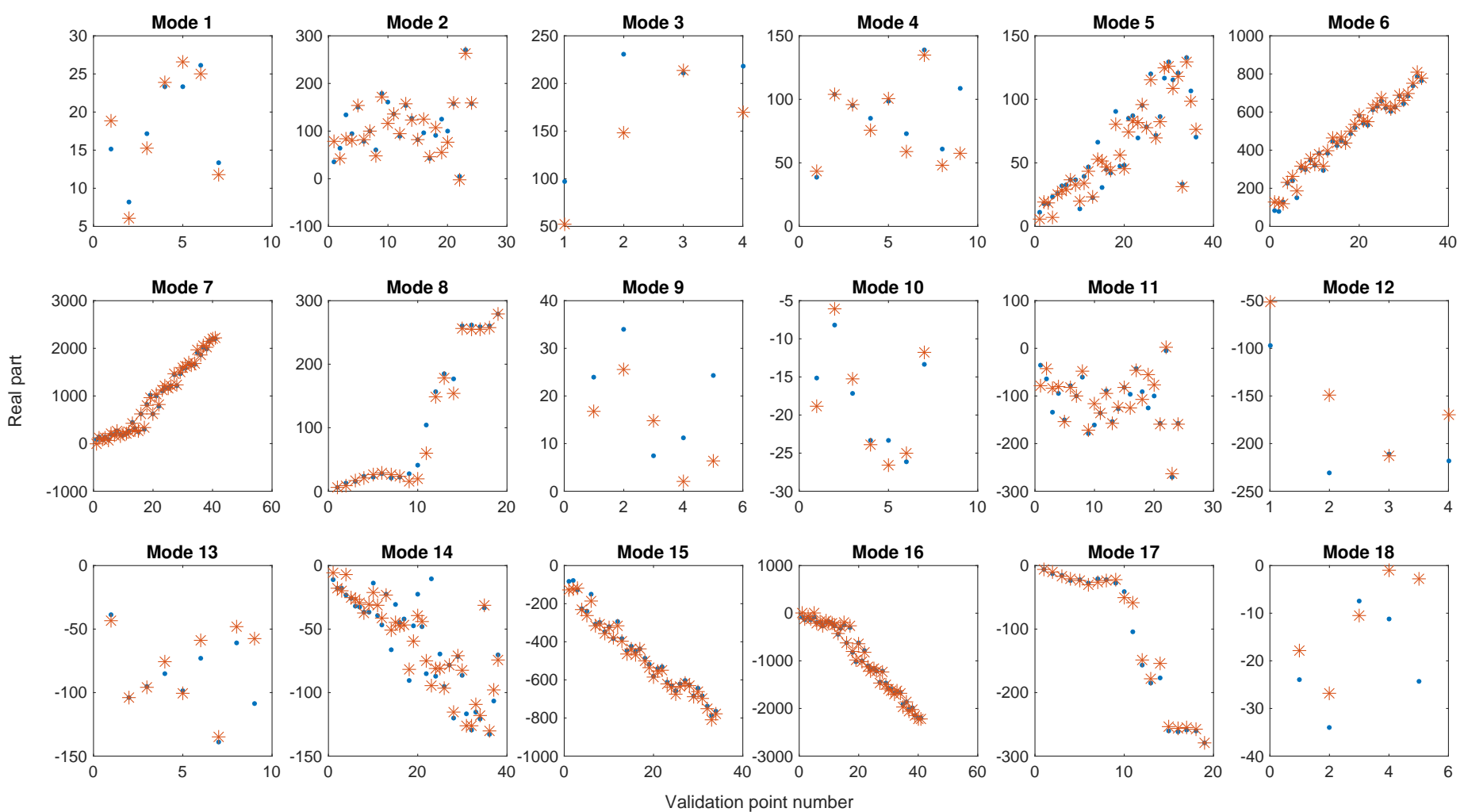

(a)
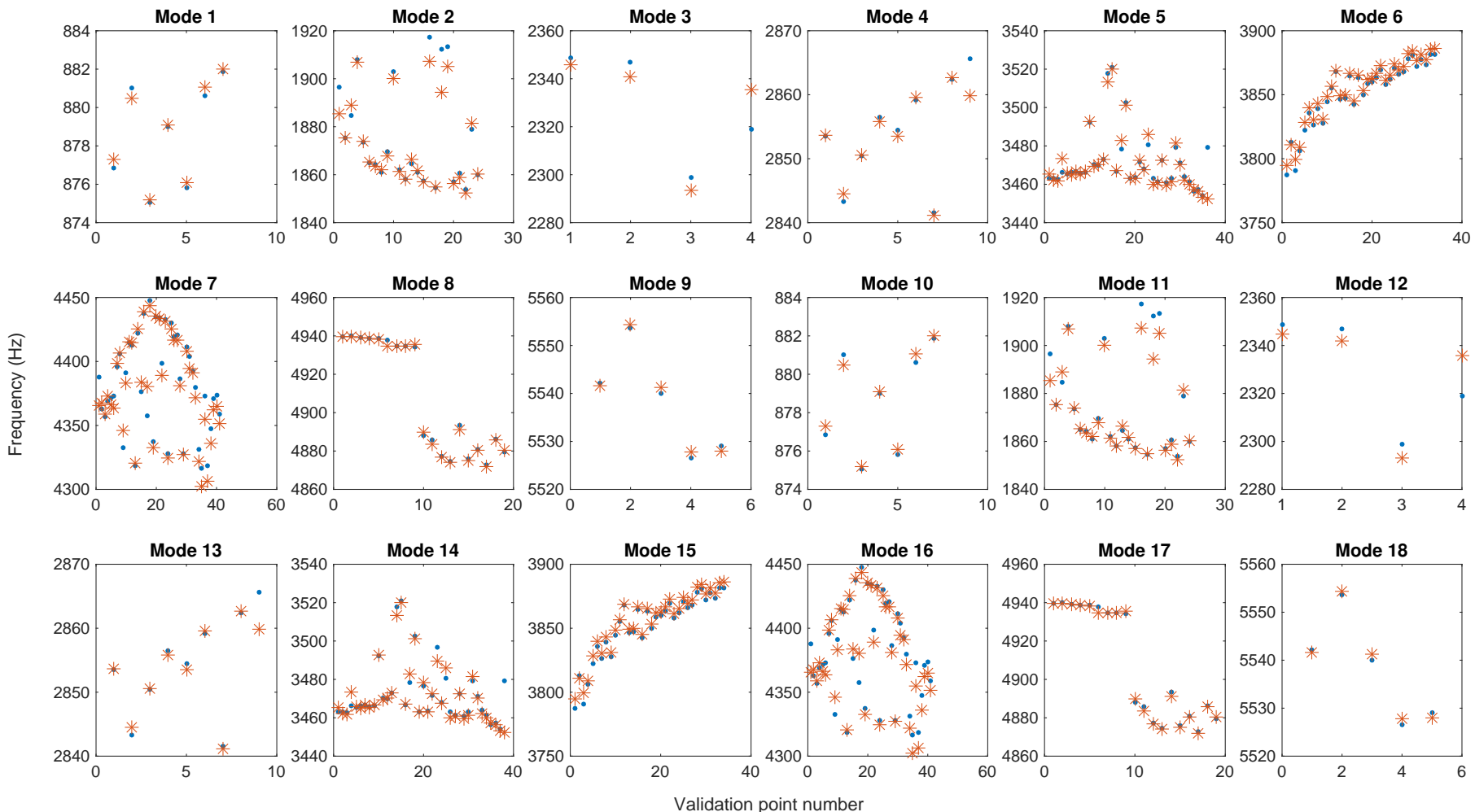

(b)

Figure 7: Comparison of the real parts (a) and the frequencies (b) of the different modes : kriging prediction $(*)$ and reference $(\bullet)$ 

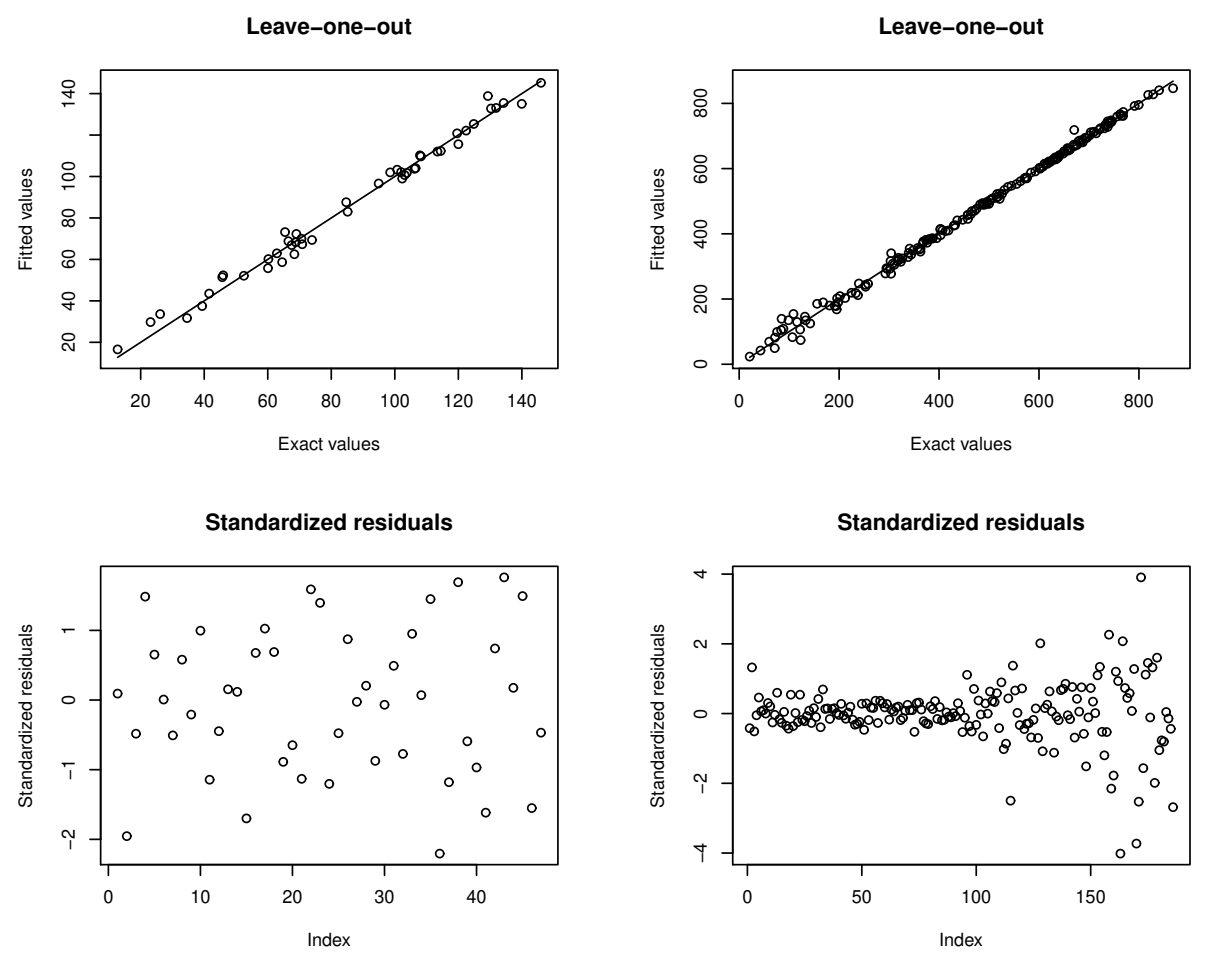

Normal QQ-plot of standardized residuals

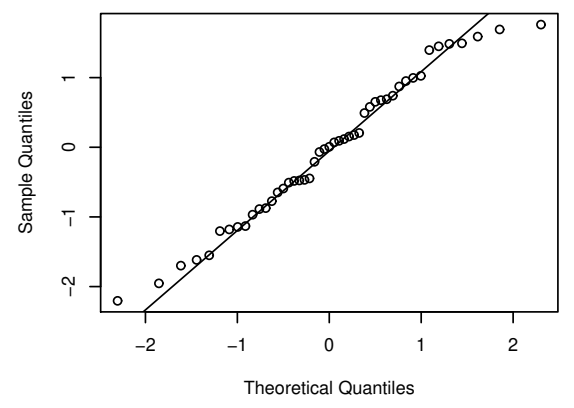

(a)

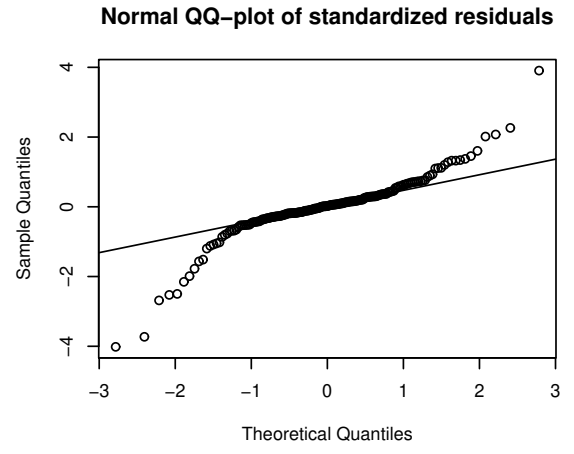

(b)

Figure 8: Validation of kriging meta-models for mode 4 (a) and mode 6 (b) - Leave-one-out validation (top) standardize residuals (middle) - Quantiles comparison (bottom)

the prediction of $\mathcal{M}_{\sim i}$ at $x_{i}$ is compared to the reference value from the $\mathrm{ED}$, namely $y_{i}$. If the meta-model is of high quality of prediction, then it is expected to have $\mathcal{M}_{\sim i}\left(x_{i}\right)=y_{i}$. This comparison is performed for each point of the ED used to create the meta-models, it is illustrated on the top graphics of Figure 8. This figure gives the scatterplot of predicted values via the LOO strategy (i.e. the circles in the plot) versus the true values. We expect the points to be on the line $y=x$ (i.e. the line in the plot) which means the fitted values are equal to the exact values. For the validation of kriging meta-models for mode 4 and mode 6 , whether there are few or many points, the fitted values are extremely close to the exact values which is highly satisfactory. The second indicator is the observation of the standardized residuals computed in the LOO process, they are given in the middle graphics of Figure 8.

$$
\frac{y_{i}-\mathcal{M}_{\sim i}\left(x_{i}\right)}{\sigma_{\sim i}\left(x_{i}\right)}
$$

where $y_{i}$ is the exact output value at point $i . \mathcal{M}_{\sim i}\left(x_{i}\right)$ defines the kriging prediction at point $i$ when the point $i$ is removed of the ED and $\sigma_{\sim i}\left(x_{i}\right)$ the associated kriging standard deviation. Here, in both cases (i.e. for kriging meta-models of mode 4 and mode 6), the standard deviations remain low (inferior to 3 in absolute value), which is satisfactory. The last indicator consists in the comparison of the quantiles of the kriging meta-model to the theoretical quantiles (i.e. the corresponding quantiles from a standard normal distribution). 
Indeed, the kriging method consists in approximating the output with a Gaussian process, but it is important to validate this assumption. For this reason, the quantiles of the process of each kriging meta-model are then compared to the theoretical quantiles of a Gaussian process. This is performed by using the classical Normal Quantile-Quantile plot graph (Normal QQ-plot), as illustrated on the bottom graphics of Figures 8(a,b). For the reader comprehension, the general QQ-plot is a graphical technique for determining if two data sets come from populations with a common distribution. In the present study, the Normal QQ-plot when both sets of quantiles come from normal distributions is used. So, if the errors of the predictions from their true values are normally distributed, it is expected that the quantiles of the kriging meta-models (i.e. the circles in the plot) should be equals to the theoretical ones (i.e. the line $y=x$ in the plot) or at least lie roughly along this line. For the two illustrative cases presented here, it is verified here.

Based on the proposed methodology for the meta-model validation, all the meta-models created have been validated using these different indicators. It is important to note that this preliminary analysis is a critical and necessary step for an engineering study. This makes it possible to validate the relevance and robustness of the meta-models, and thus to be able to trust the prediction of the squeal propensity from the latter.

\subsection{Squeal prediction}

Once the meta-models are created and validated it is possible to use them to predict the squeal propensity of the brake system. As an illustration, the evolution of the real parts and of the frequencies of five modes, namely modes 2, 4, 5, 6 and 8, are given Figure 9 and Figure 10 respectively. The evolutions versus the two masses $M_{1}$ and $M_{2}$ are given for three different values of $\mu$, namely $0.3,0.5$ and 0.7 . White zones correspond to the area for which the real part of the eigenvalues is equal to zero, and it only reflects that the system is stable. The meta-model does not predict the frequency evolution for this zone due to the fact that no instability is present. Therefore Figure 10 gives only the evolution of the frequency of unstable modes.

First of all, it is very clear that the results on the squeal propensity of the brake system are not intuitive. The impact of adding these two masses can lead to changes in the brake's behaviour that are sometimes complex and require a fine analysis of the results and the role of these additional masses. Indeed, it is observed that the influence of the masses $M_{1}$ and $M_{2}$ on the stability of the system depends of the considered mode and of the value of the friction coefficient $\mu$. Increasing the friction coefficient may lead to an increase, decrease or no significant change of the instability area (see and compare for example the squeal propensity for modes 2,6 and 8).

More specifically some comments can be made to illustrate the complexity of the results and the analysis that can be carried out on the influence of these two additional masses:

- In the case of the mode 2, the stability area is more complex and is made of several distinct zones. Increasing the friction coefficient $\mu$ induces an expansion of the unstable area. Moreover, the mass $M_{2}$ has a low influence on the real part of the system whereas the increase of $M_{1}$ tends to stabilize the system. Hence, when the value of the mass $M_{1}$ is superior to $150 \mathrm{~g}$ and $\mu=0.3$, then the system is always stable. When $\mu=0.7$, if $M_{1}$ is superior to $220 \mathrm{~g}$, the mode is always unstable;

- The mode 4 is almost always stable when $\mu=0.3$. Increasing the friction coefficient increases the instability area for mode 4: the mode becomes unstable when $M_{1}$ and $M_{2}$ increase simultaneously. For this mode, the location of the unstable zone is always obtained when both the additional masses are important;

- In the case of the mode 5 , two stable zones are detected for $\mu=0.3$. For $\mu=0.7$ the system is unstable. The "most unstable zone" (i.e. the design area where the real part is the highest) is located for a low value of $M_{1}$.

- the mode 6 is always unstable (i.e. for $\mu=0.3, \mu=0.5$ and $\mu=0.7$ ). The "most unstable zone" is not the same for these three cases. For $\mu=0.3$, this zone corresponds to a high value of the mass $M_{1}$ while it is identified for a zero mass $M_{1}$ in the cases of $\mu=0.5$ and $\mu=0.7$;

- the mode 8 is always stable when $\mu=0.3$ and always unstable when $\mu=0.5$. For $\mu=0.7$, the mode has a stable and an unstable area. However, the maximum value of the real part is observed for $\mu=0.7$ (and not for $\mu=0.5$ ). The addition of a mass $M_{1}$ tends to increase the real part of the unstable mode. For $\mu=0.7$, if the mass $M_{2}$ is always superior to $150 \mathrm{~g}$, then the mode is always stable. The design area where the mode is the most unstable for $\mu=0.5$ and $\mu=0.7$, in the sense of the highest real part, remains the same: it is located around the point $M_{1}=225 \mathrm{~g}$ and $M_{2}=20$;

All these remarks demonstrate the different sensitivities of each mode to the modifications of the two additional masses $M_{1}$ and $M_{2}$. If in some cases, the addition of mass has a stabilization effect, in other cases it can 


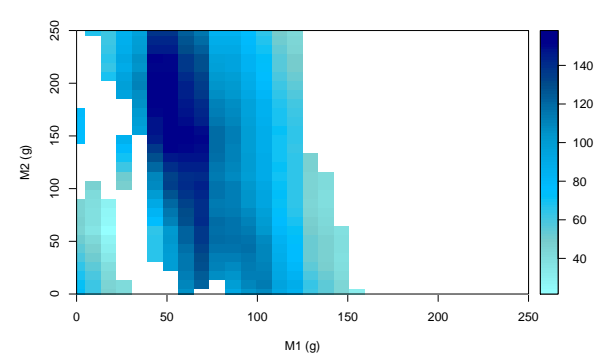

(a) Mode $2-\mu=0.3$

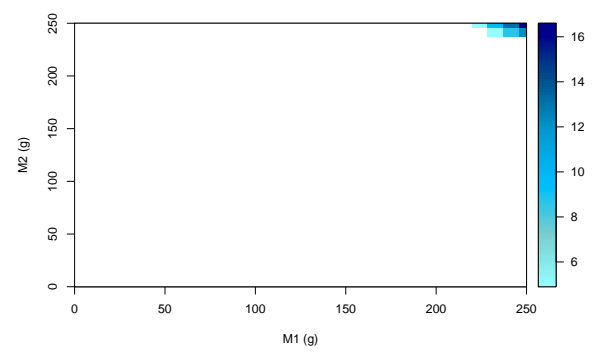

(d) Mode $4-\mu=0.3$

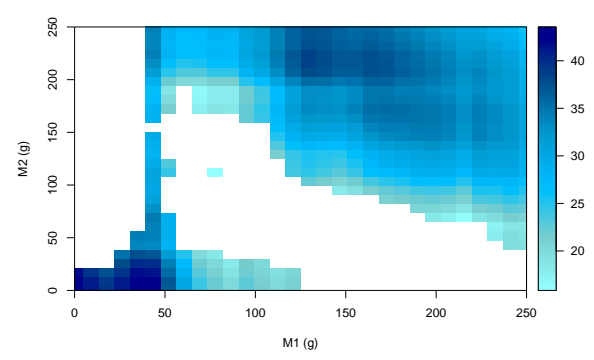

(g) Mode $5-\mu=0.3$

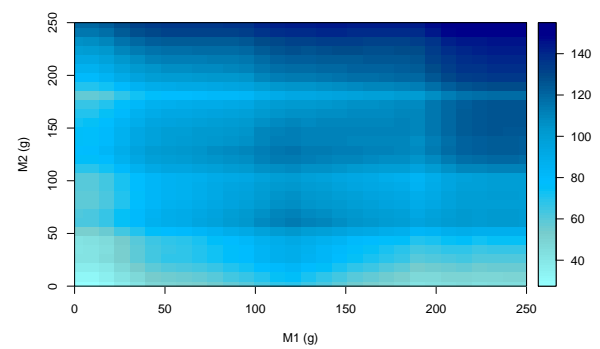

(j) Mode $6-\mu=0.3$

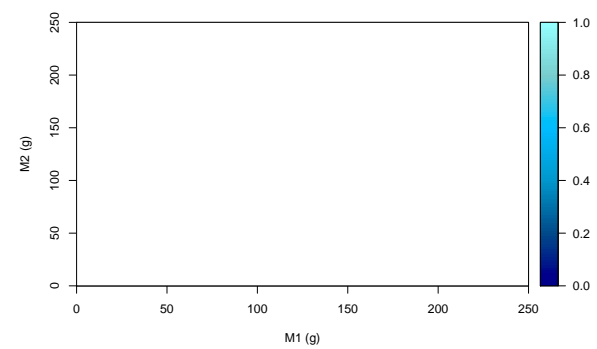

(m) Mode $8-\mu=0.3$

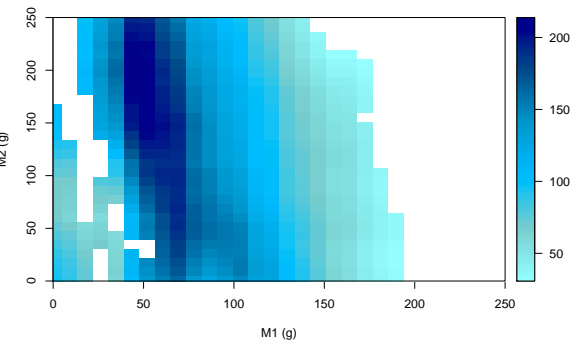

(b) Mode $2-\mu=0.5$

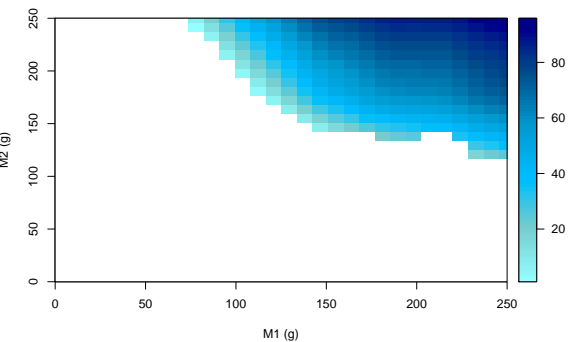

(e) Mode $4-\mu=0.5$

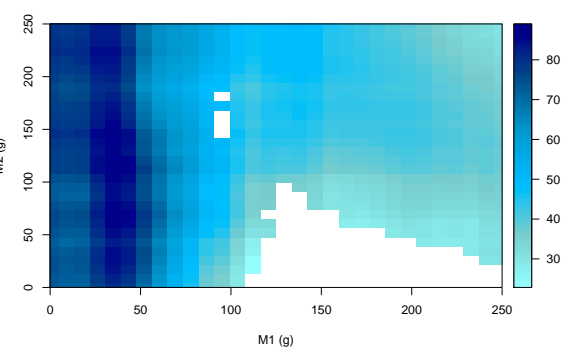

(h) Mode $5-\mu=0.5$

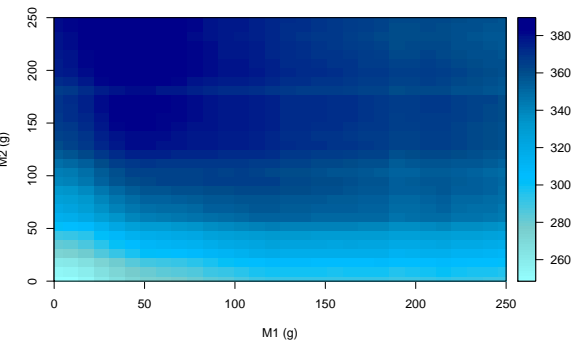

(k) Mode $6-\mu=0.5$

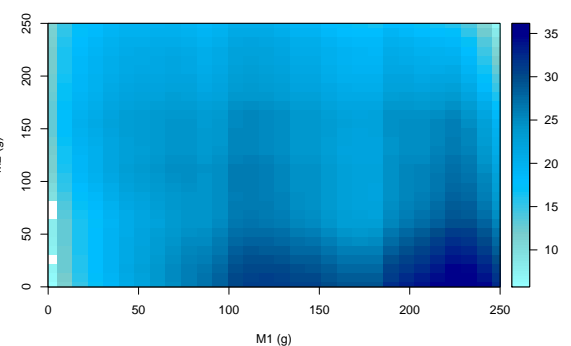

(n) Mode $8-\mu=0.5$

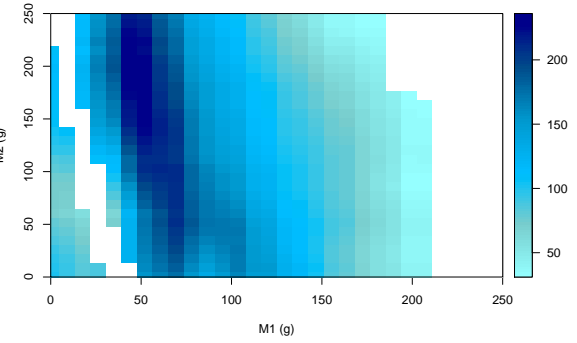

(c) Mode $2-\mu=0.7$

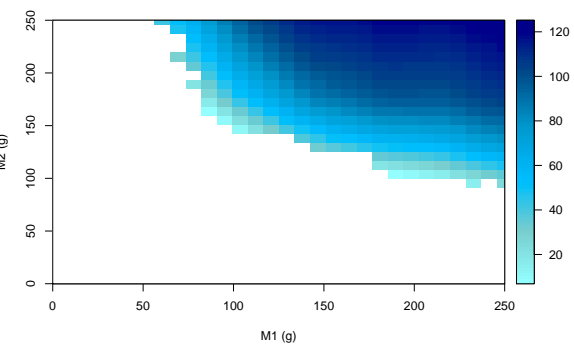

(f) Mode $4-\mu=0.7$

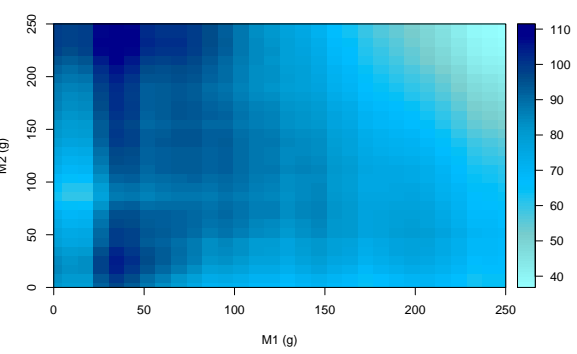

(i) Mode $5-\mu=0.7$

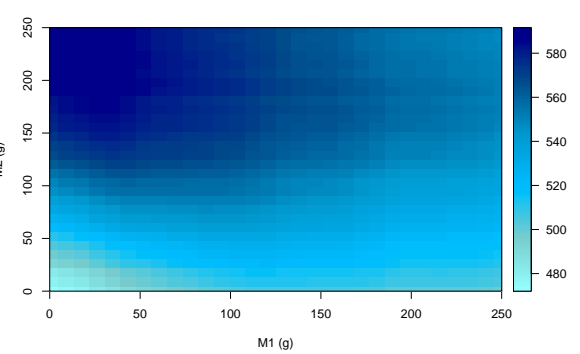

(l) Mode $6-\mu=0.7$

Figure 9: Evolution of real parts of the 1st, 3nd, 4th, 6th and 8th unstable modes versus $M_{1}$ and $M_{2}$ for different values of the friction coefficient $\mu$ : from left to right: $\mu=0.3, \mu=0.5$ and $\mu=0.7$ - In white, the areas for which the system is stable (i.e. null real part). 


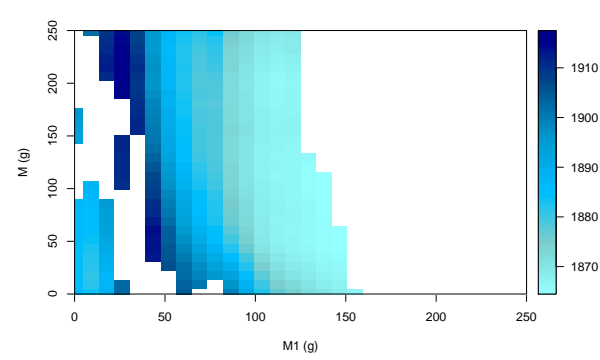

(a) Mode $2-\mu=0.3$

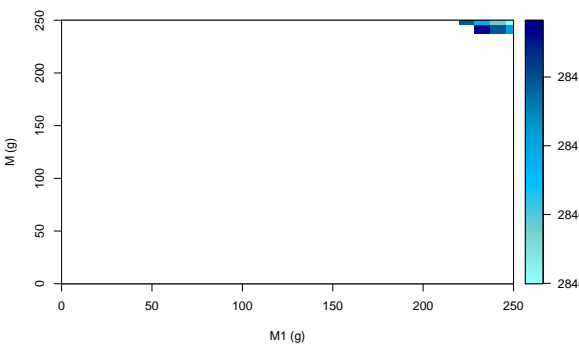

(d) Mode $4-\mu=0.3$

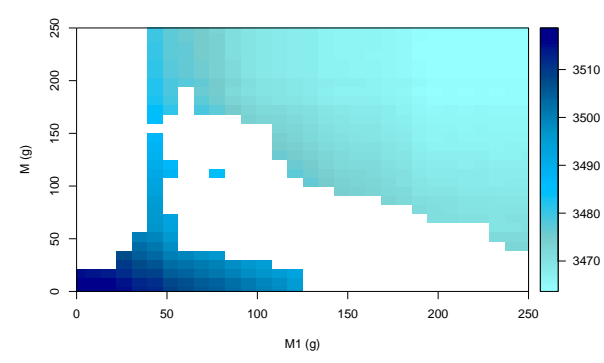

(g) Mode $5-\mu=0.3$

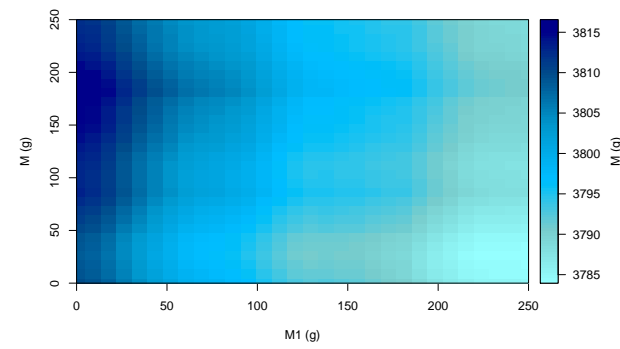

(j) Mode $6-\mu=0.3$

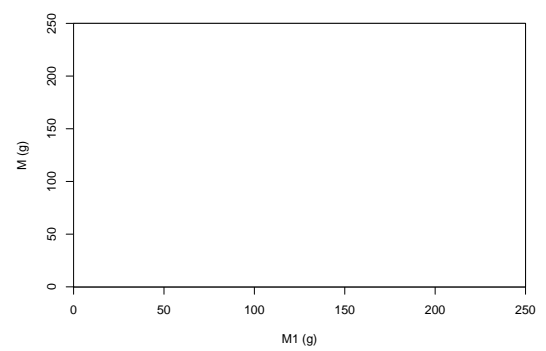

(m) Mode $8-\mu=0.3$

g) Mode $5-\mu=0.3$

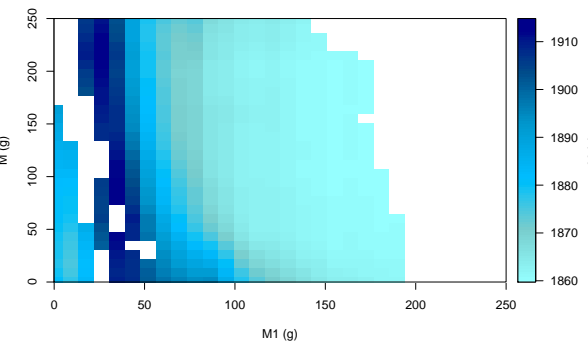

(b) Mode $2-\mu=0.5$

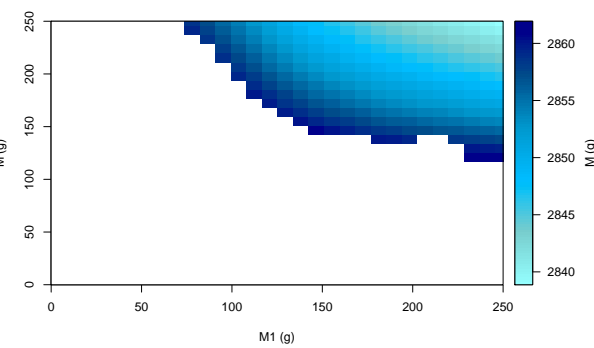

(e) Mode $4-\mu=0.5$

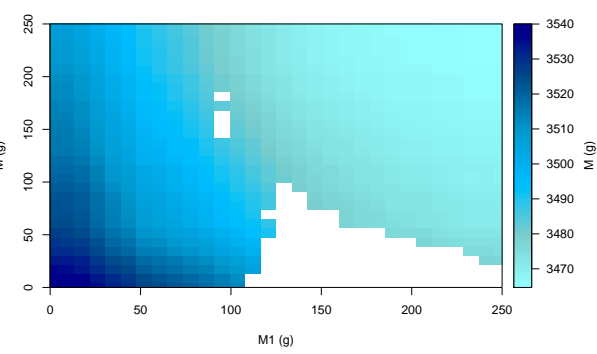

(h) Mode $5-\mu=0.5$

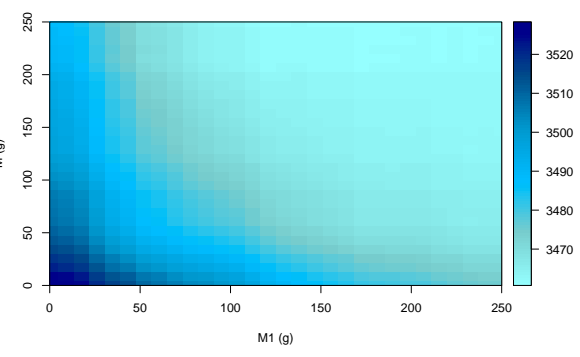

(i) Mode $5-\mu=0.7$

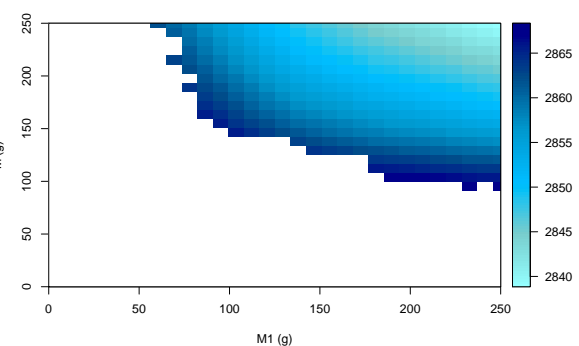

(f) Mode $4-\mu=0.7$ (c) Mode $2-\mu=0.7$

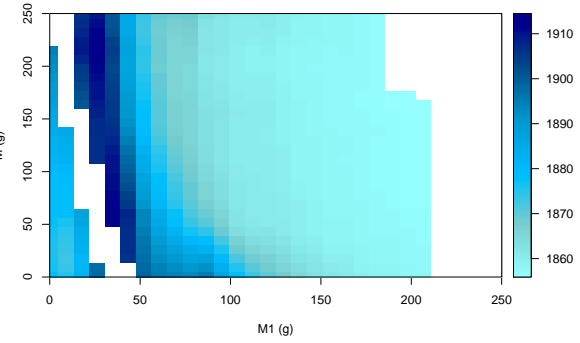

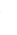

(k) Mode $6-\mu=0.5$
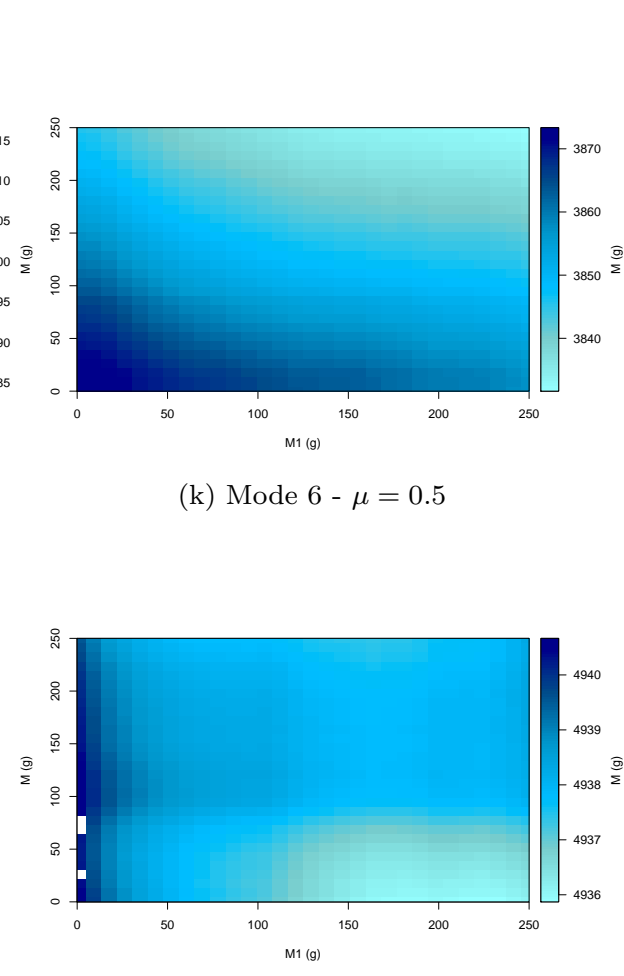

(n) Mode $8-\mu=0.5$

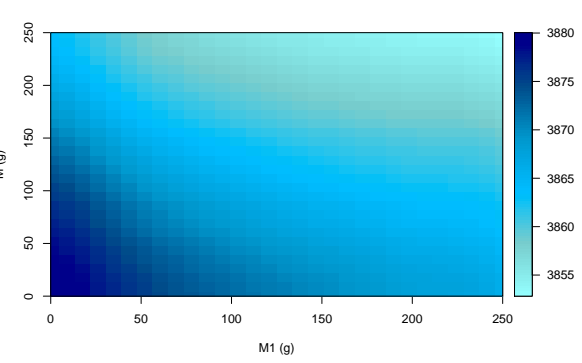

(1) Mode $6-\mu=0.7$

Figure 10: Evolution of the frequency of the 1st, 3nd, 4th, 6th and 8th unstable modes versus $M_{1}$ and $M_{2}$ for different values of the friction coefficient $\mu$ : from left to right: $\mu=0.3, \mu=0.5$ and $\mu=0.7$ - In white, the areas for which the system is stable (i.e. null real part) and so frequencies are not estimated 
destabilize some modes, and the location of the "most unstable zone" (i.e. the design area where the real part is the highest) is not the same for all the unstable modes. It is worth noting that the phenomenon of mode coupling (i.e. its generation and evolution), as well as the two modes involved in a coalescence of modes can also be impacted by such structural modifications. This fact has not be observed in this particular case.

Similar conclusions can be made by analysing the frequency evolution of each unstable mode. The addition of mass $M_{1}$ and/or $M_{2}$ modifies more or less the frequencies of each mode. Some modes are more impacted by a modification of $M_{1}$, as the mode 2 , whereas other are more impacted by a modification of both $M_{1}$ and $M_{2}$, as the mode 4. Finally, in some cases the influence of the masses is highly dependent to the value of the friction coefficient $\mu$. For example, for the mode 6 , when $\mu=0.3$, the frequency is sensitive to a modification of $M_{1}$ whereas when $\mu=0.7$, the two masses are influential.

These observations can be translated by the Sobol indices [29]. The first order indices are defined as follows:

$$
S_{i}=\frac{\operatorname{Var}\left[E\left[Y \mid X_{i}\right]\right]}{\operatorname{Var}[Y]}
$$

and the total order indices are given by:

$$
S T_{i}=1-\frac{\operatorname{Var}\left[E\left[Y \mid X_{\sim i}\right]\right]}{\operatorname{Var}[Y]}
$$

where $\operatorname{Var}(Y)$ is the variance of the aleatory variable $Y$ defined by $f\left(X_{1}, \ldots, X_{d}\right)$ where $\left(X_{1}, \ldots, X_{d}\right)$ are $d$ aleatory variables. And $X_{\tilde{i}}=X_{1}, X_{2}, \ldots, X_{i-1}, X_{i+1}, \ldots, X_{d}$. Figure 11 illustrates the results for the real parts and the frequencies. First order indices are given in light grey and are an indicator of the influence of the parameter lonely. Total indices are given in dark grey and correspond to the influence of the associated parameters and its coupling with other parameters. A low Sobol indices means the influence of the parameter is low, a high Sobol indices (i.e. almost equals to 1) means the influence is strong. These kinds of analysis give indications about the influence of the different parameters and their coupling effect. Hence, the real part of the mode 6 only depends of the friction coefficient $\mu$ and is not sensitive to masses $M_{1}$ and $M_{2}$. In the case of the mode 2, the first order indices related to $M_{1}$ is largely higher than the indices of $\mu$ and $M_{2}$. It means the real part of the mode 2 is more sensitive to a modification of the mass $M_{1}$. This is confirmed by results displayed Figure 9(d-f) where variations of $\mu$ and $M_{2}$ have a low impact on the real part whereas a variation of $M_{1}$ has a larger influence on it. In the case of the mode 4, the three first order indices are relatively close. Indeed, they are all located between 0.19 and 0.4 , meaning all the parameters have an influence on the real part. However, the difference between first order indices and total indices is quite large, so the effects are mostly coupled. The influence of the different parameters on the frequencies are often different of the influence on the real parts. For example, for the mode 1 the friction coefficient $\mu$ is the parameter with the larger impact on the real part whereas its influence on the frequency is almost null. The latter is more sensitive to variations of $M_{1}$ and $M_{2}$.

In conclusion, all these results on squeal propensity demonstrate the complex influence of the different parameters on the system stability. It proves the necessity to perform deep studies on the prediction of squeal propensity for an industrial brake system. The unstable zones generated by the different modes are not trivial. From a practical and industrial point of view, this clearly highlights the difficulty for an engineer to find intuitively the set of parameters that decreases the squeal occurrences of a brake system. Moreover, the large number of parameter to deal with and the complexity of the problem highlight the advantage of the strategy presented here, based on a meta-modelling method. This strategy results in obtaining the influence of the various parameters for a controlled numerical cost. Indeed, less than 250 CEA are required here, whereas $100^{3}$ CEA would have been required to get the same level of precision with a parametric study.

\subsection{Extension to the inclusion of uncertainty propagation}

As previously explained in Section 2.3, the friction coefficient $\mu$ can be described by a distribution law instead of a deterministic fixed value. This choice aims to propose a more realistic modelling of the coefficient of friction. The problem posed is then to be able to provide an answer on the impact of the friction law chosen on the squeal occurrences.

Because the numerical cost of the meta-models is low, it is possible to performed Monte Carlo Samplings (MCS) on the meta-models to propagate the uncertainty associated to $\mu$. Hence, three different probability laws are considered for the friction coefficient: two beta laws of shape parameters $(2,8)$ and $(8,2)$, denoted $\mathrm{B}(2,8)$ and $\mathrm{B}(8,2)$ respectively, and an uniform distribution. The three distribution laws are displayed Figure 12 on the left column. A grid of size $(10 \times 10)$ is considered for masses $M_{1}$ and $M_{2}$ and for each couple $\left(M_{1}, M_{2}\right)$, 1000 Latin Hypercube Sampling (LHS) on $\mu$ are considered. This is done for each law on $\mu$. Two kind of results are considered: the mean number of instabilities and the mean of the sum of the positive real parts, displayed on Figure 12 in the middle and right column respectively. 

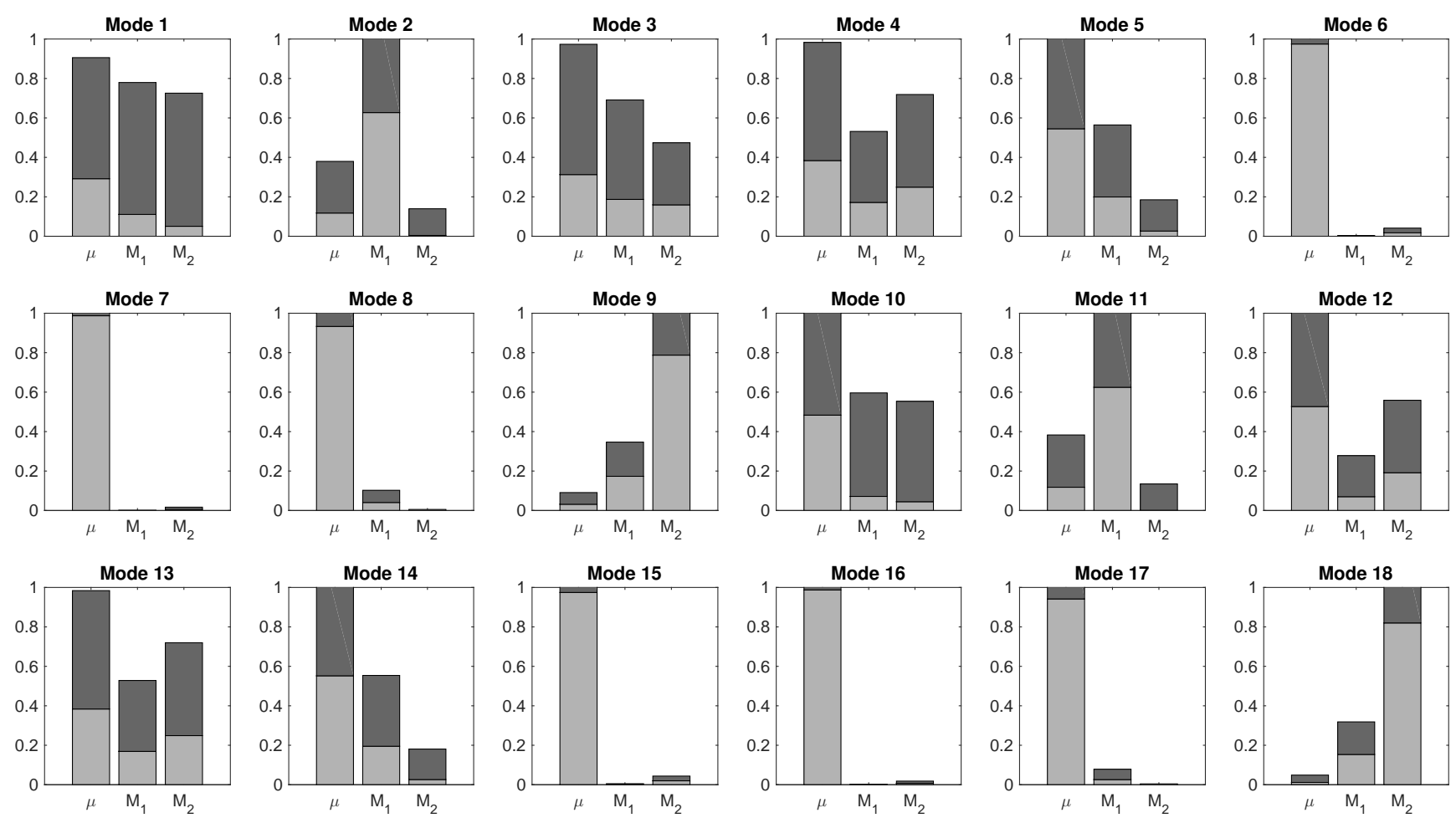

(a)
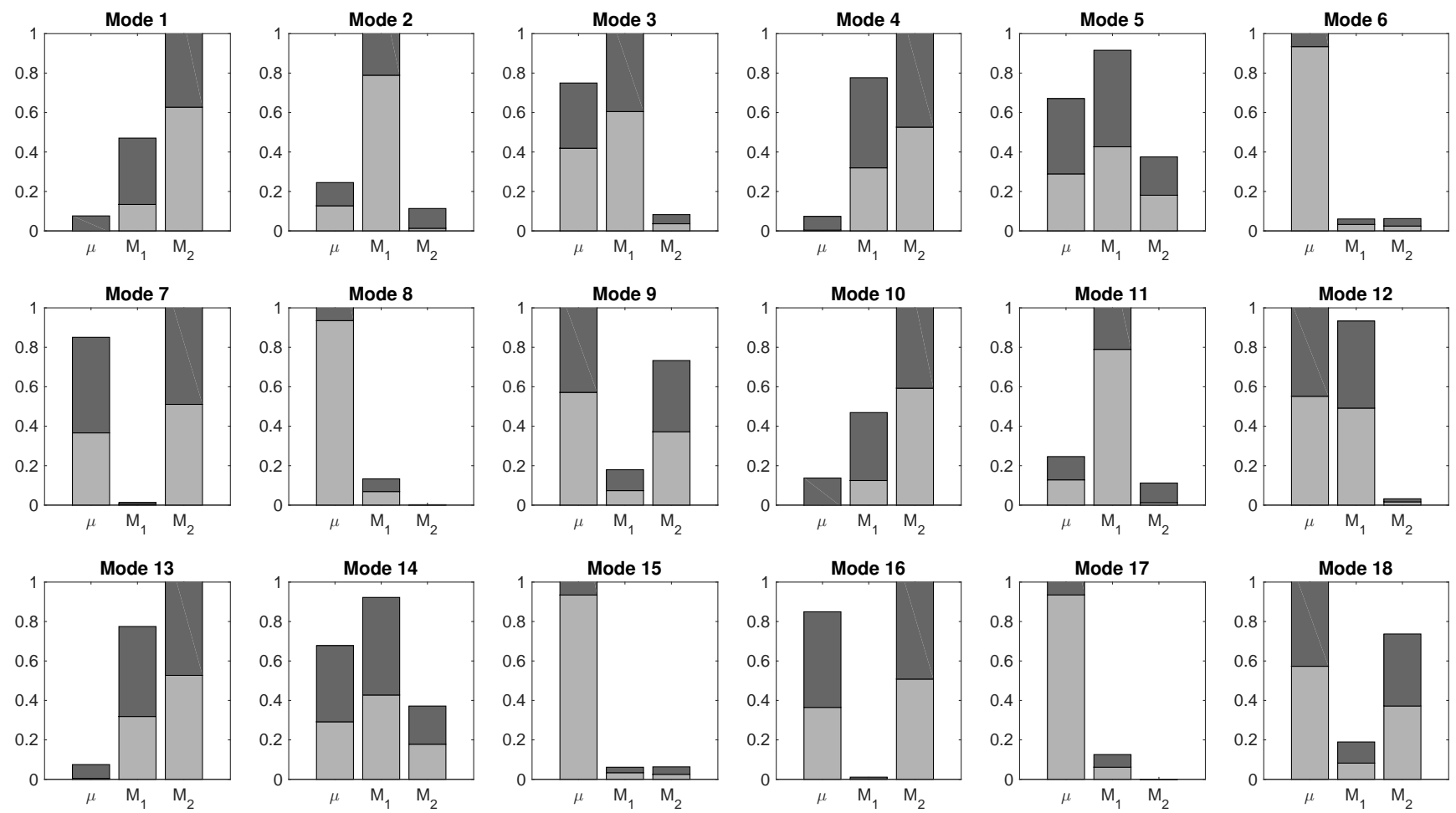

(b)

Figure 11: Sobol indices of real parts (a) and frequency (b) of the different modes determined from the metamodels $\mathcal{M}_{a}$ and $\mathcal{M}_{\omega}$ respectively - Light grey: first order indices - Dark grey: total indices 

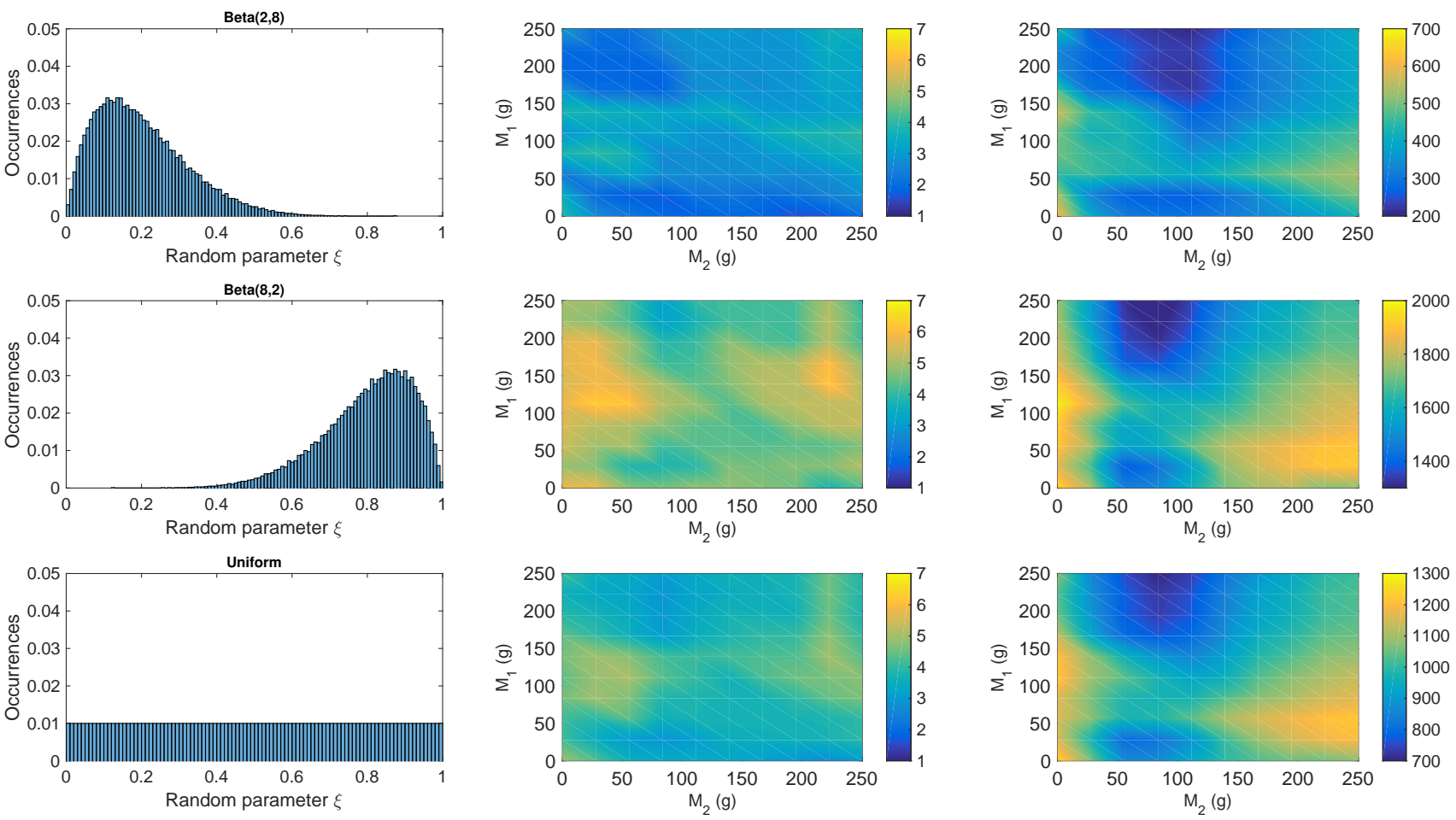

Figure 12: Probability law (left), mean number of instabilities (middle) and mean of the sum of the positive real parts (right) for the different probability laws: $\mathrm{B}(2,8)$ (top) - $\mathrm{B}(8,2)$ (middle) - Uniform (bottom)

First, the high impact of the probability law chosen for $\mu$ can be noted. Hence, for a $\mathrm{B}(2,8)$ law, the system has in mean, a maximum of 4 instabilities and a minimum of 1.5 instabilities. Whereas with a $\mathrm{B}(8,2)$ law, the system has, in mean, a maximum of 6 instabilities and a minimum of 3 instabilities. The influence on the mean of the sum of the positive real parts is also important. In the case of the $B(2,8)$ law, the mean varies between 200 and 600 whereas with a $B(8,2)$ law, it varies between 1250 and 2000. Moreover, the variations of the mean number of instabilities and the mean of the sum of the positive real parts also depends of the chosen distribution. For example, in the case of a $B(2,8)$ law, the mean number of unstable modes is minimal and equal to 1.5 if $M_{1}=50 \mathrm{~g}$ and $M_{2}=200 \mathrm{~g}$. If it is a $B(8,2)$ law or a uniform law, this minimum is reached for $M_{1}=100 \mathrm{~g}$ and $M_{2}=225 \mathrm{~g}$. Similarly, for the mean of the sum of the positive real parts, the minimum is reached for $M_{1}=110 \mathrm{~g}$ and $M_{2}=250 \mathrm{~g}$ when $\mu$ follows a $B(2,8)$ law and variations around this points are low. In the case of a $B(8,2)$ distribution or a uniform distribution, this minimum is located at $M_{1}=80 \mathrm{~g}$ and $M_{2}=250 \mathrm{~g}$ with more important evolutions around this point.

These results coupling with the results presented on Figures 9 and 10 also show the difficulty to select the best design for the masses in order to suppress or to reduce squeal in the current system. It is recalled that it is necessary to determine the non-linear dynamic response of the brake system (i.e. the determination of self-excited vibrations) in order to select the best design to reduce brake squeal. The proposed methodology based on a kriging strategy only provides the optimal design versus the occurrences of brake squeal.

As a conclusion, these results illustrate the importance to identify as soon as possible and the more precisely as possible the probability laws of the physical parameters considered as uncertain during a squeal study. This study also shows the interest of the use of meta-models that allow to perform additional parametric studies with a reasonable calculation cost.

\section{Conclusion}

The present study proposed the use of the kriging methodology to predict the stability behaviour of an automotive brake system subjected to a lot of potential unstable modes. The main objective is to illustrate the relevance of this approach for predicting the evolution of each unstable modes of the brake system with respect to structural modifications of the system. 
Hence, the CEA procedure is used to predict the stability of the finite element model of an automotive brake system. Three uncertain parameters are considered in the present study, namely the friction coefficient and two small masses added to the caliper. The addition of two small masses corresponds to a classical choice in the field of the automotive industry for structural modifications used in the final phase of a brake design to avoid squeal noise.

The first part of the study highlights the difficulty to track the evolution of unstable modes at the coalescence point, so the eigenvalues can be tracked only when their real parts are non zero and so the meta-models are built only on this area. Once the meta-models are validated, it is possible to predict the squeal behaviour of the brake system.

Results show the high impact of the two additional small masses on the squeal propensity of each mode. Above all, it appears that each mode has a completely different behaviour versus squeal occurrences and the impact of these additional masses depends of the considered mode. This result can be explained by the fact that adding small masses can drastically modify the mode shapes of the caliper which is often involved in the mode coupling phenomena and so on the stability behaviour of the brake system. Thereby, all the proposed analyses highlight the complex behaviour of an automotive brake system that has many unstable modes and the difficulty to identify the best design in regard to brake squeal. The results obtained tend to suggest that the kriging method can be considered to identify the best design with a lower number of simulations and/or with a larger number of parameters.

Finally, an extension of the proposed approach with the inclusion of uncertainty propagation is performed to assess a robust analysis of the propensity of brake squeal. Results show the importance of a correct identification of the input distribution laws before performing uncertainty propagation, the latter ones having a very strong impact on the results of brake squeal.

\section{Acknowledgments}

This work was achieved within PSA Peugeot Citroën Stellab program - OpenLab Vibro-Acoustic- Tribology@Lyon(VAT@Lyon).

J.-J. Sinou acknowledges the support of the Institut Universitaire de France.

\section{References}

[1] R.A. Ibrahim. Friction-induced vibration, chatter, squeal, and chaos-part ii: dynamics and modeling. Applied Mechanics Reviews, 47(7):227-253, 1994.

[2] R.T. Spurr. A theory of brake squeal. Proceedings of the Institution of Mechanical Engineers: Automobile Division, 15(1):33$52,1961$.

[3] J. Hultén. Brake squeal-a self-exciting mechanism with constant friction. Technical report, SAE Technical Paper, 1993.

[4] N. Hoffmann, M. Fischer, R. Allgaier, and L. Gaul. A minimal model for studying properties of the mode-coupling type instability in friction induced oscillations. Mechanics Research Communications, 29(4):197-205, 2002.

[5] J.-J. Sinou. Transient non-linear dynamic analysis of automotive disc brake squeal - on the need to consider both stability and non-linear analysis. Mechanics Research Communications, 37:96-105, 2010.

[6] A. Loyer, J-J. Sinou, O. Chiello, and X. Lorang. Study of nonlinear behaviors and modal reductions for friction destabilized systems. application to an elastic layer. Journal of Sound and Vibration, 331(5):1011-1041, 2012.

[7] N. Coudeyras, S. Nacivet, and J-J. Sinou. A new treatment for predicting the self-excited vibrations of nonlinear systems with frictional interfaces: the constrained harmonic balance method, with application to disc brake squeal,. Journal of Sound and Vibration, 319:1175-1199, 2009.

[8] H. Ouyang, W. Nack, Y. Yuan, and F. Chen. Numerical analysis of automotive disc brake squeal: a review. International Journal of Vehicle Noise and Vibration, 1(3-4):207-231, 2005.

[9] A.F. D'souza and A.H. Dweib. Self-excited vibrations induced by dry friction, part 2: Stability and limit-cycle analysis. Journal of Sound and Vibration, 137(2):177-190, 1990.

[10] T. Tison, A. Heussaff, F. Massa, I. Turpin, and R.F. Nunes. Improvement in the predictivity of squeal simulations: Uncertainty and robustness. Journal of Sound and Vibration, 333(15):3394-3412, 2014.

[11] A. Culla and F. Massi. Uncertainty model for contact instability prediction. The Journal of the Acoustical Society of America, 126(3):1111-1119, 2009.

[12] T. Butlin and J. Woodhouse. Friction-induced vibration: Quantifying sensitivity and uncertainty. Journal of Sound and Vibration, 329(5):509-526, 2010.

[13] E. Sarrouy, O. Dessombz, and J-J. Sinou. Piecewise polynomial chaos expansion with an application to brake squeal of a linear brake system. Journal of Sound and Vibration, 332(3):577-594, 2013.

[14] H. Lü and D. Yu. Brake squeal reduction of vehicle disc brake system with interval parameters by uncertain optimization. Journal of Sound and Vibration, 333(26):7313-7325, 2014.

[15] H. Lü and D. Yu. Optimization design of a disc brake system with hybrid uncertainties. Advances in Engineering Software, 98:112-122, 2016

[16] M. Treimer, B. Allert, K. Dylla, and G. Müller. Uncertainty quantification applied to the mode coupling phenomenon. Journal of Sound and Vibration, 388:171-187, 2017.

[17] A. Nobari, H. Ouyang, and P. Bannister. Uncertainty quantification of squeal instability via surrogate modelling. Mechanical Systems and Signal Processing, 60:887-908, 2015. 
[18] L. Nechak, F. Gillot, S. Besset, and J-J. Sinou. Sensitivity analysis and kriging based models for robust stability analysis of brake systems. Mechanics Research Communications, 69:136-145, 2015.

[19] E. Denimal, J-J.Sinou, S. Nacivet, and L. Nechak. Squeal analysis based on the effect and determination of the most influential contacts between the different components of an automotive brake system. International Journal of Mechanical Sciences, 151:192-213, 2019.

[20] S. Oberst and J.C.Ss Lai. Statistical analysis of brake squeal noise. Journal of Sound and Vibration, 330(12):2978-2994, 2011.

[21] O. Roustant, D. Ginsbourger, and Y. Deville. Dicekriging, diceoptim: Two r packages for the analysis of computer experiments by kriging-based metamodeling and optimization. 2012.

[22] S.N. Lophaven, H.B. Nielsen, and J. Søndergaard. DACE: a Matlab kriging toolbox, volume 2. Citeseer, 2002.

[23] F. Bachoc. Parametric estimation of covariance function in Gaussian-process based Kriging models. Application to uncertainty quantification for computer experiments. PhD thesis, Université Paris-Diderot-Paris VII, 2013.

[24] C. Lataniotis, S. Marelli, and B. Sudret. Uqlab user manual-kriging (gaussian process modelling). Report UQLab-V0, pages 9-105, 2015.

[25] E. Denimal, L. Nechak, J-J Sinou, and S. Nacivet. Kriging surrogate models for predicting the complex eigenvalues of mechanical systems subjected to friction-induced vibration. Shock and Vibration, 2016, 2016.

[26] E. Balmes. Parametric families of reduced finite element models. theory and applications. Mechanical Systems and Signal Processing, 10(4):381-394, 1996.

[27] P-Y. Tourneau, P. Bousquet, F. Mercier, and M. Klein. Application of scatter analyses in satellite development. Spacecraft structures and mechanical testing, pages 341-353, 1994.

[28] A. Forrester, A. Sobester, and A. Keane. Engineering design via surrogate modelling: a practical guide. John Wiley \& Sons, 2008.

[29] I.M. Sobol. Global sensitivity indices for nonlinear mathematical models and their monte carlo estimates. Mathematics and computers in simulation, 55(1-3):271-280, 2001. 